\title{
Toddalolactone Protects Against Osteoarthritis by Ameliorating Chondrocyte Inflammation and Suppressing Osteoclastogenesis
}

\section{Yiming Xu}

Shanghai General Hospital Department of Orthopaedics: Shanghai Jiaotong University First People's Hospital Department of Orthopaedics

\section{Song Xue}

First Affiliated Hospital of Anhui Medical University

\section{Tian Zhang}

Yueqing City People's Hospital: Wenzhou Medical College Affiliated Yueqing Hospital

\section{Xinmeng Jin}

Shanghai General Hospital Department of Orthopaedics: Shanghai Jiaotong University First People's Hospital Department of Orthopaedics

\section{Cong Wang}

Shanghai General Hospital Department of Orthopaedics: Shanghai Jiaotong University First People's Hospital Department of Orthopaedics

\section{Haiming Lu}

Shanghai General Hospital Department of Orthopaedics: Shanghai Jiaotong University First People's Hospital Department of Orthopaedics

\section{Yiming Zhong}

Shanghai General Hospital Department of Orthopaedics: Shanghai Jiaotong University First People's Hospital Department of Orthopaedics

\section{Hongjie Chen}

Shanghai General Hospital Department of Orthopaedics: Shanghai Jiaotong University First People's Hospital Department of Orthopaedics

\section{Libo Zhu}

Shanghai General Hospital Department of Orthopaedics: Shanghai Jiaotong University First People's Hospital Department of Orthopaedics

\section{Jinzhong Ma}

Shanghai General Hospital Department of Orthopaedics: Shanghai Jiaotong University First People's Hospital Department of Orthopaedics weilin Sang ( $\sim$ sangweilin001@163.com )

Shanghai General Hospital Department of Orthopaedics: Shanghai Jiaotong University First People's Hospital Department of Orthopaedics 


\section{Research Article}

Keywords: Toddalolactone, cartilage, inflammation, osteoclastogenesis, osteoarthritis

Posted Date: December 28th, 2021

DOI: https://doi.org/10.21203/rs.3.rs-1170145/v1

License: (c) (i) This work is licensed under a Creative Commons Attribution 4.0 International License. Read Full License

Version of Record: A version of this preprint was published at Chinese Medicine on February 5th, 2022. See the published version at https://doi.org/10.1186/s13020-022-00576-w. 


\section{Abstract}

Background: Osteoarthritis $(\mathrm{OA})$ is widely recognized as the most common chronic joint disease accompanied by progressive cartilage and subchondral bone damage. Toddalolactone (TOD), a natural compound extracted from Toddalia asiatica (L.) Lam., has been widely used in the treatment of stroke, rheumatoid arthritis, and oedema. Nevertheless, what TOD acts as in the pathogenesis and progression of OA hasn't been reported. In this investigation, we have aimed to determine how TOD affects OA in vitro and in vivo.

Methods: LPS $(10 \mu \mathrm{g} / \mathrm{ml})$ was employed to induce chondrocyte inflammation or RANKL to induce osteoclast differentiation in bone marrow derived macrophages (BMMs); Meanwhile, the effects of TOD on chondrocyte inflammation and osteoclast differentiation were evaluated. Anterior cruciate ligament transection (ACLT) was performed to develop an OA animal model and study the effects of TOD.

Results: We found that TOD inhibited the expression of inflammatory and catabolic mediators ( IL-6, IL-8, TNF-a, MMP2, MMP9, and MMP13) in LPS-treated chondrocytes in vitro. Furthermore, TOD was proven to inhibit RANKL-induced-osteoclastogenesis and inhibit the expression of marker genes (NFATC1 and cfos). Our data also confirmed that TOD suppressed the destruction of articular cartilage and osteoclastogenesis by inhibiting the activation of the NF-KB and MAPK signalling pathways. In the ACLT mouse model, we found that TOD attenuated the ACLT-induced cartilage erosion and inhibited bone resorption.

Conclusions: These results showed that TOD can be adopted as a potential therapeutic agent for OA.

\section{Background}

Osteoarthritis (OA) is widely recognized as the most common chronic joint disease(1). As the population ages and with obesity on the rise, the incidence of $O A$ is increasing. According to the Rotterdam study(2), $53 \%$ of women and $33 \%$ of men over the age of 80 had OA of the knee. OA often causes in the destruction of the articular cartilage and subchondral bone sclerosis, resulting in inflammation and subsequent stiffness, swelling, and difficulty in moving the joint $(3,4)$. Pain and loss of function seriously reduce patients' quality of life, and the high cost of treatment greatly increases the social and economic burden(5). Considering the complex pathogenesis and high incidence of $O A$, it is necessary to explore more treatment methods.

OA was thought to be just a "wear and tear" disease(6). Currently, our understanding of OA has changed dramatically. OA can have a significant impact on articular cartilage, leading to serious degeneration of articular cartilage in the course of the disease, which often leads to low-grade inflammatory features of the cartilage $(7,8)$. After inflammatory stimulation, the chondrocytes transform into a degraded phenotype, which not only activates nuclear factor-kappaB (NF-kB) and mitogen-activated protein kinase (MAPK) signaling pathways but also leads to the secretion of a series of inflammatory factors, comprising matrix metalloproteinases (MMPs) and a disintegrin and metalloproteinases with 
thrombospondin motifs (ADAMTS)(9-11). OA can gradually lead to cellular changes, structural defects, and dysfunction of the entire interarticular compartment $(12,13)$. It is defined as a whole-joint disease, which causes the rupture of articular cartilage and causes subchondral sclerosis $(9,14)$. The subchondral bone is located at the epiphyseal layer below the articular cartilage(15). Bone homeostasis depends on osteoblast-mediated bone formation and osteoclast-mediated bone resorption $(16,17)$. The balance between bone formation and bone resorption is important for subchondral bone stability and shock absorption and support in normal joints $(18,19)$. During $O A$, disruption of joint homeostasis often leads to excessive activation of osteoclasts, causing increased bone resorption and subchondral densification, thereby contributing to the progression of $O A(20)$. Therefore, it is of great significance to find drugs that can prevent cartilage degeneration and activate osteoclasts for the treatment of OA.

It has been considered that chondrosarcoma SW1353 cells are suitable cellular model for OA research(21-23). IL-1ßstimulation results in a decrease in type II collagen and aggrecan levels, which are the two main organic components of articular cartilage(9). This demonstrates that SW1353 cells have cytological characteristics of chondrocytes. RAW246.7 exhibits biological behavior similar with that of the osteoclast precursor, which can be transformed to osteoclasts by appropriate cytokine stimulation(24, 25). These two types of cells are easy to culture and grow rapidly and thus are used to study the potential anti-chondrocyte inflammation and anti-osteoclastogenesis effect of traditional Chinese medicines. Over the past few decades, compounds extracted from traditional Chinese medicinal plants have shown strong anti-inflammatory effects and may hold the key to treating $O A(26,27)$. Toddalolactone (TOD) is a natural compound extracted from Toddalia asiatica (L.) Lam., and has been used for treating various chronic diseases for a long time(28). A previous study had reported that TOD has anti-embolism, hemostatic and anti-fibrotic effects(29). TOD is a traditional Chinese medicine distributed in Guangxi, Yunnan, and Guizhou provinces, and is broadly adopted in treating diseases like stroke, rheumatoid arthritis, and oedema(30). Nevertheless, what TOD acts as in the pathogenesis and progression of OA has not yet been reported.

In the investigation, we have investigated how TOD affects anti-chondrocyte inflammation and inhibition of osteoclastogenesis, and further explored the possibility of TOD as a new potential drug for OA.

\section{Materials And Approaches}

\section{Reagents and cell lines}

Toddalolactone (MF: C16H2006, MW:308.33) came from MedChem Express (Shanghai, China). Dimethyl sulfoxide (DMSO), Penicillin, streptomycin, and tartrate-resistant acid phosphate (TRAP) came from Sigma-Aldrich (USA). RIPA lysis buffer came from Beyotime (Shanghai). Lipopolysaccharides (LPS) and macrophage colony stimulating factor (M-CSF) came from Peprotech (USA). RANKL came from R\&D Systems (USA). Primary bone marrow-derived macrophages (BMMs) were separated from the bone marrow of 5-week-old male C57BL/6 mice. SW1353 and RAW264.7 cell lines came from the Cell Bank of Chinese Academy of Sciences (Shanghai). 


\section{Cell viability}

The cell counting Kit-8 (CCK-8) analysis (Dojindo, Japan) was employed for assessing the toxicity of TOD. SW1353, BMMs and RAW264.7 cells were cultured in 96-well plates with a density of $7 \times 10^{3}$ cells/well. After pre-treatment with gradient concentrations $(0,0.5,1,5,10,20$, or $40 \mu \mathrm{M})$ of TOD for the indicated time or being left without treatment, $10 \mu \mathrm{L} \mathrm{CCK}-8$ was added to the 96 -well plate and incubated at $37^{\circ} \mathrm{C}$ for 3 hours. Then we measured the optical density (OD) at $450 \mathrm{~nm}$ with a microplate photometer.

\section{Flow cytometry analysis}

SW1353 cells were inoculated on 6-well plates $\left(3.0 \times 10^{5} /\right.$ well) and treated with gradient concentrations of $\operatorname{TOD}(0,0.5,1,10,20$, or $40 \mu \mathrm{M})$ for 24 hours. The cells were gathered after treatment and stained with Annexin V-PE and 7-AAD assay kits on basis of the manufacturer's instructions.

\section{Western blotting}

After treatment under different conditions, total proteins were collected from the cells adopting ice-cold RIPA lysis buffer. BCA protein assay (Beyotime, China) were adopted for measuring relative protein concentrations. After separation on SDS-PAGE gels, the protein was transferred to the PVDF membrane, which were sealed with $5 \%$ skimmed milk for 2 hours. Then the membrane was incubated overnight with a first antibody at $4^{\circ} \mathrm{C}$. Enhanced chemiluminescence was employed for visualizing the bands. The following antibodies were used: PARP (9532, Cell Signalling Technology), C-PARP (5625, Cell Signalling Technology), Bcl-xl (2764, Cell Signalling Technology), Bcl-2 (3498, Cell Signalling Technology), Bax (5023, Cell Signalling Technology), $\beta$-Tubulin (10094-1-AP, Proteintech), Trap (ab52750, Abcam), Ctsk (ab187647, Abcam), c-fos (Ab222699, Abcam), NFATc1 (Ab253477, Abcam), GAPDH (60004-1-lg, Proteintech), P-P65 (3033, Cell Signalling Technology), P65 (8242, Cell Signalling Technology), IkBa (4814, Cell Signalling Technology), P-ERK (4370, Cell Signalling Technology), ERK (4695, Cell Signalling Technology), P-P38 (4511, Cell Signalling Technology), P38 (8690, Cell Signalling Technology), P-JNK (9255, Cell Signalling Technology), JNK (9252, Cell Signalling Technology), and H3 (17168-1-AP, Proteintech).

\section{Real-time PCR analysis}

After a specific treatment, total mRNA was extracted from various cells with TRIZOL. These RNA was reversely transcribed into cDNA adopting PrimeScript RT Master Mix (TaKaRa, Dalian, China). Real-time PCR was performed with TB Green Premix Ex Tap on basis of the manufacturer's instructions. The primers employed are listed below: IL-6, forward 5'-CAACCTGAACCTTCCAAAGATG-3', reverse 5'ACCTCAAACTCCAAAAGACCAG-3'; IL-8, forward 5'-GAGAGTGATTGAGAGTGGACCAC-3', reverse 5'CACAACCCTCTGCACCCAGTTT-3'; TNF-a, forward 5'-CACTTCGAAACCTGGGATTCAG-3', reverse 5'GGTCTCCAGATTCCAGATGTCAG-3'; MMP2, forward 5'-AGCGAGTGGATGCCGCCTTTAA-3', reverse 5'CATTCCAGGCATCTGCGATGAG-3'; MMP9, forward 5'-GCCACTACTGTGCCTTTGAGTC-3', reverse 5'CCCTCAGAGAATCGCCAGTACT-3'; MMP13, forward 5'-CCTTGATGCCATTACCAGTCTCC-3', reverse 5'AAACAGCTCCGCATCAACCTGC-3'; NFATC1, forward 5'-GGTGCCTTTTGCGAGCAGTATC-3', reverse 5'- 
CGTATGGACCAGAATGTGACGG-3'; Trap, forward 5'-GCGACCATTGTTAGCCACATACG-3', reverse 5'CGTTGATGTCGCACAGAGGGAT-3'; Ctr, forward 5'-CTGGGATGGCTGGATGTG-3', reverse 5'-

TGCTGTCAGGGTGTCTAAAC-3'; Ctsk, forward 5'-AGCAGAACGGAGGCATTGACTC-3', reverse 5'CCCTCTGCATTTAGCTGCCTTTG-3'; DC-STAMP, forward 5'-TTTGCCGCTGTGGACTATCTGC-3', reverse 5'GCAGAATCATGGACGACTCCTTG-3'; VATPase D2, forward 5'-ACGGTGATGTCACAGCAGACGT-3', reverse 5'-CTCTGGATAGAGCCTGCCGCA-3'.

\section{ELISA}

We collected supernatants from different cells after specific treatment. Then MMPs and pro-inflammatory cytokines were detected with ELISA kits (Bangyi, Shanghai, China) on the basis of the prepared standard curve.

In vitro osteoclast differentiation assay

The BMMs were inoculated into 96 -well plates at a density of $8 \times 10^{3}$ cells/well and adhered to adhere to the wall for 2 days. Various TOD contents $(0,5,10$, and $20 \mu \mathrm{M})$ were dissolved in the medium to test its effect on osteoclast differentiation. The osteoclastogenic medium was altered every two days until the fifth day. Four percent PFA was used to fix the cultured BMMs, and the formation of osteoclasts was observed by Trap staining (Solarbio, Beijing, China).

\section{Bone resorption pit assay}

Round calf bone slices measuring $8 \mathrm{~mm}$ (STX0012A, Thousand Sunrise, China) were autoclaved and then placed in 96-well plates. The BMMs were inoculated on calf bone slices at a density of $8 \times 10^{3}$ cells/well, and osteoclast differentiation was induced in a-MEM containing medium supplemented with $25 \mathrm{ng} / \mathrm{ml} \mathrm{M}$-CSF and $50 \mathrm{ng} / \mathrm{ml}$ RANKL. Different TOD contents $(0,5,10$, and $20 \mu \mathrm{M})$ were dissolved in the medium to test their effects on osteoclast differentiation. After 5 days, the calf bone slices were removed from the 96-well plates, and the cells on the surface were brushed off.

\section{Nuclear and cytoplasmic extraction}

After treatment of the SW1353 and BMMs cells with TOD under different conditions, we extracted nuclear and cytoplasmic proteins for observing the expression of P65 and P-P65. The nuclear and cytoplasmic proteins were extracted applying nuclear and cytoplasmic protein separation kits under the manufacturer's instructions

\section{Immunofluorescence staining}

The treated cells were immobilised with 4\% PFA at room temperature for 40 minutes and incubated employing $1 \%$ Triton for 15 minutes for membranes penetrations. Then, cells were then blocked with $0.2 \%$ BSA for 1 hour and cultured adopting a primary antibody at $4^{\circ} \mathrm{C}$ overnight. After rinsed three times applying sterile PBS, the cells were visualized adopting Alexa Fluor-conjugated secondary antibodies for 15 min. After staining the nuclei employing DAPI for 10 minutes, the cells were rinsed three times 
applying sterile PBS and observed under a fluorescence microscope (Olympus FluoViewTM FV1000, Tokyo, Japan).

\section{Construction of Mouse OA models}

To study the therapeutic effects of TOD on the mouse knee joint, we surgically constructed a mouse osteoarthritis model through anterior cruciate ligament transection (ACLT) of the knee joint. In brief, 20, 8week-old, male C57BL/ 6 mice came from the Animal Center of the Chinese Academy of Sciences and were raised at the Experimental Animal Department of Shanghai General Hospital. The 20 mice were divided into four groups with five mice in each group: sham, ACLT + PBS, ACLT + Low TOD, and ACLT + High TOD groups. Specifically, in the ACLT operation group, the mice were anesthetized through intraperitoneal injection of $4 \%$ chloral hydrate, and ACLT was performed using a needle in the left knee without skin incision. The anterior drawer detection was used for testing the effect of surgery. Mice of the sham group $(n=5)$ were not operated on. Mice of the ACLT + PBS group $(n=5)$ underwent ACLT, and PBS (30 mg/kg) was injected intraperitoneally. Mice of the ACLT + Low TOD underwent ACLT and we injected TOD $(6 \mathrm{mg} / \mathrm{kg})$ intraperitoneally. Mice of the ACLT + High TOD group underwent ACLT, and we injected TOD $(12 \mathrm{mg} / \mathrm{kg})$ intraperitoneally. All the experiments were carried out in accordance with the prescribed animal welfare procedures, and this investigation was approved by the Ethics Committee of Shanghai General Hospital (\#2021AW064).

\section{Histological assessments}

The left knees of the mice were fixed in 4\% PFA for 24 hours at 6 weeks postoperatively. All the knees were then decalcified adopting 10\% EDTA for 1 month and embedded in paraffin. Subsequently the knees were sliced into 4- $\mu \mathrm{m}$-thick sections and stained with H\&E, Safranin O/Fast Green, and Toluidine Blue.

\section{TUNEL staining}

Apoptotic cells in the articular cartilage were detected by terminal deoxynucleotide transferase dUTP staining under the manufacturer's instructions. The quantity of TUNEL-positive cells was quantified.

\section{Statistical analysis}

All data exhibited as the average \pm SD of three independent experiments. SPSS software was used for data analysis. One-way ANOVA was employed for statistic analyses $\left({ }^{*}, \#\right.$ indicates $p<0.05$, ,,$\# \#$ indicates $p$ $<0.01$, ns indicates not significant).

\section{Results}

TOD inhibits the expression of inflammatory and catabolic mediators in LPS-treated chondrocytes in vitro.

Figure 1A shows the molecular structure of TOD. For investigating the toxic impact of TOD on chondrocytes, we used the CCK8 assay kit, western blot assay and flow cytometry analysis to detect the effect of gradient concentrations $(0,0,51,5,10,20$, and $40 \mu \mathrm{M})$ of TOD on chondrocytes. Fig. 1B-1D 
shows that no significant cytotoxicity of TOD on chondrocytes was observed. Our next experiment used concentrations in this range. LPS are commonly used to induce arthritis in vitro conditions(31). Results of the RT-PCR analysis revealed that LPS stimulation caused significant increase in pro-inflammatory cytokine and MMP levels in chondrocytes, and TOD significantly reduced the increase in LPS-induced inflammatory cytokines, and MMP levels (Fig. 1E). ELISA revealed that TOD reduced the protein levels of MMP2, MMP9, MMP13, IL-6, and TNF-a secreted by chondrocytes after treatment with LPS (Fig. 1F). These finding demonstrated that TOD inhibits the expression of inflammatory and catabolic mediators in LPS-treated chondrocytes.

TOD inhibits RANKL-induced osteoclastogenesis, formation of F-actin ring, and bone resorption in vitro.

The CCK8 assay kit was conducted for detecting how TOD affected the cell survivability of BMMs and RAW264.7. Fig. 2A and 2B present that TOD exhibited no cytotoxic effects in either BMMs or RAW264.7 cells at contents as high as $20 \mu \mathrm{M}$. Therefore, for the following experiments, no more than $20 \mu \mathrm{M}$ of TOD doses was used. An in vitro osteoclastogenesis mode is required for exploring how TOD affects osteoclastogenesis(32). When RANKL the cells were stimulated adopting various TOD contents $(0,5,10$, and $20 \mu \mathrm{m} / \mathrm{ml}$ ) for $5 \mathrm{~d}$, the number of osteoclasts formed gradually decreased (Fig. 2C). Fig. 2D presents the quantification of the area occupied by the osteoclasts and number of osteoclasts, which showed the same results as Fig. 2C. Furthermore, to investigate the stage of osteoclast formation, we supplemented $20 \mu \mathrm{M}$ TOD to the osteoclastogenic medium at three different phases: d1 to d3 (early phase), d3 to d5 (late phase) and d1 to d5 (all phases). Fig. 2E shows that compared to the late phase, early TOD intervention significantly inhibited osteoclastogenesis. Fig. $2 \mathrm{~F}$ performs the quantification of the area occupied by osteoclasts and the number of osteoclasts in Fig. 2E. Bone resorption assay are often used to test osteoclast formation. Then the same amount of BMMs was inoculated onto the calf bone slices. Fig. $2 \mathrm{G}$ shows that with increasing concentrations of TOD, osteoclastogenesis was significantly inhibited. The quantification of resorption area and numbers of osteoclast rings were showed in Fig. $2 \mathrm{H}$. Fig. $2 \mathrm{I}-2 \mathrm{~J}$ show the same trends in Fig. 2E-2F. Immunofluorescence staining showed that the increasing concentration of TOD gradually suppressed the actin ring formation, and the inhibitory effect was concentration dependent and better when TOD was added in the early phase (Fig. 3A-3D). These results suggests that TOD significantly inhibited osteoclastogenesis, especially in the early phase.

\section{TOD inhibits RANKL-induced osteoclast-related gene expression in vitro.}

For further studying the mechanism of TOD in inhibiting osteoclastogenesis, mRNA expression of osteoclast marker genes (NFATc1, Trap, Ctr, Ctsk, DC-STAMP, and VATPase D2) were measured through RT-PCR. The outcomes showed that TOD reduced the expression of osteoclast formation marker genes in a dose-dependently manner (Fig. 4A). Outcomes of western blots analysis exhibited that TOD significantly suppressed the expression of c-Fos and NFATc1 (Fig. 4B). Fig. 4C shows the western blot bands in Fig. 4B. These findings indicate that TOD suppressed RANKL-induced osteoclast-related gene expression in vitro. 
As a major metabolic pathway in OA, NF-KB signalling is involved in LPS-induced inflammation and RANKL-induced osteoclast formation. After stimulation, the activated NF-KB molecule triggers the expression of a series of genes that induce the destruction of articular cartilage and active osteoclast formation. The NF-KB signaling path is considered as an essential regulator of cartilage destruction and bone remodeling in $\mathrm{OA}(18,33,34)$. SW1353 cells were stimulated with LPS or LPS+TOD for various time periods $(0,3,6,9$, and 12 hours) to investigate the mechanism of TOD on chondrocyte inflammation. Meanwhile, to study the mechanism of TOD action on osteoclast formation, BMMs were stimulated employing RANKL or RANKL+TOD for various times $(0,5,15,30$, and 60 minutes). Western blotting was adopted for detecting the expression of the NF-KB signalling pathway. Fig. 5A and Fig. 6A show that unlike in the LPS group or the RANKL group, TOD treatment greatly lessened the expression of P-P65, PERK and P-P38, and inhibited the degradation of IKBa, which are the key genes associated with NF-KB signalling. Fig. 5B and Fig. 6B show the quantification of the western blot banks. We then extracted nuclear and cytoplasmic proteins to detect the translocation of p65. Fig. 5C-5Dand Fig. 6C-6D confirm that TOD suppressed the translocation of P65 into the nucleus. Immunofluorescence staining in (Fig. 5E and Fig. 6E) intuitively revealed that the nuclear translocation of p65 was reduced after TOD treatment. The MAPK signalling pathway has been reported to be significantly associated with osteoarthritis cartilage damage and bone remodeling $(35,36)$. In this investigation, TOD obviously inhibited the phosphorylation of JNK in SW1353 and BMMs (Fig. 5A and Fig. 6A). Our data indicated both NF-KB and MAPK signaling paths were suppressed, which suggested that TOD may restrain the destruction of articular cartilage and osteoclastogenesis by inhibiting the activation of the NF-KB and MAPK signaling paths.

\section{TOD prevents against ACLT-induced cartilage destruction and inhibits osteoclastogenesis in vivo.}

For further studying the protective impact of TOD on osteoarthritis in vivo, we first constructed a mouse osteoarthritis model through ACLT of the knee joint, which is a classical model for the study of OA. Based on the previous description, the mice were classified into four groups with five C57BL/6J mice in each group. H\&E staining and Safranin O/Fast Green staining in Fig. 7A revealed cartilage erosion in the ACLT + PBS group, unlike in the Sham group, whereas ACLT + TOD treatment attenuated this erosion. The OARSI scoring standard was employed for quantifying the structural alterations in tibial plateau cartilage (Fig. 7D). Each sample in the treatment group was independently evaluated blind by two experienced experimentalists. The two observers also agreed on the results. TUNEL DAPI/FITC staining was adopted for measuring chondrocyte apoptosis. The quantity of TUNEL-positive cells in the ACLT+PBS group increased significantly, whereas the quantity of TUNEL-positive cells decreased in the ACLT + TOD group (Fig. 7A), in comparison to the Sham group. The number of apoptotic cells were counted (Fig. 7E). Furthermore, the expression level of Collagen II in cartilage was measured by immunohistochemical staining. As shown in Fig. 7B, ACLT + PBS lessened the expression of Collagen II in comparison to that in the sham group, whereas TOD treatment rescued these changes. Collagen II-positive cell number/total layer cell number was quantified (Fig. 7F). Furthermore, Trap staining of the subchondral trabecular bone was performed. As shown in Fig. 7C, the ACLT + PBS group exhibited an obvious rise in the quantity of Trap-positive cells in the subchondral trabecular bone and increased osteoclast activity, while the ACLT + 
TOD group attenuated the changes. In brief, these data have indicated that TOD prevents against ACLTinduced cartilage destruction and inhibits osteoclastogenesis in vivo.

\section{TOD prevents against ACLT-induced bone loss in vivo.}

Subchondral bone resorption often occurs after ACLT in mice(37). For evaluating how TOD therapeutically affects subchondral bone loss after ACLT in mice, we have used 3D reconstruction microCT to examine the structure of the subchondral bone. As shown in Fig. 8A, in the ACLT group, the loss of subchondral bone mass was significant, while intraperitoneal injection of TOD significantly inhibited bone resorption. SMI, BV/TV, BMD, Tb.N, Tb.Th, and Tb.Sp were detected on basis of the 3D reconstruction images (Fig. 8B). Furthermore, Fig. 8C showed TOD had no adverse effects on key organs and body weight in mice. In summary, these outcomes implied that TOD may be a potential therapeutic agent for OA.

\section{Discussion}

Pathological changes in OA affects the whole joint, including focal and progressive hyaline cartilage loss, and subchondral bone changes, including osteophytes and increased thickness of the bone capsule(38). Although these pathological processes may selectively affect individual articular tissues, there are close biological and physical interactions among them, and ultimately all components of the knee will interact with each other due to their close association(9). At present, the purpose of non-surgical treatment for OA is mainly to relieve patients' pain and delay the progression of $O A$; it is necessary to explore more effective drugs $(39,40)$. In this investigation, how TOD affects OA have been evaluated.

Articular cartilage has no blood vessels or nerves and consists mainly of type II Collagen and aggrecan, in addition to being made up of $70 \%$ water(41). Chondrocytes are the only cells in cartilage and undergo important phenotypic changes during the development of $\mathrm{OA}$, and are characterized by decreased secretion of type II Collagen and aggrecan, and increased secretion of inflammatory cytokines, MMPs, and $\operatorname{ADATMs}(42,43)$. These can lead to the disruption of the original homeostasis of cartilage tissue and induce the occurrence of OA. In this investigation, it has been found that TOD suppressed the LPSinduced release of matrix-degrading enzymes and pro-inflammatory cytokines at doses that do cause toxic effects in chondrocytes. These results suggested that in LPS-induced chondrocytes in vitro, TOD suppressed the expression of inflammatory and catabolic mediators.

From the perspective of chondrocytes, it is difficult to achieve good therapeutic effects in the treatment of osteoarthritis. The subchondral bone holds the keys to OA progression(44). During the development of osteoarthritis, there are significant changes in the composition and structure of the subchondral bone, including raised cortical plate thickness, lessened subchondral cancellous bone mass, changes in architecture, and bone attrition(45). These changes adversely affect the upper calcification and articular cartilage $(46,47)$. Osteoclasts are key cells involved in subchondral bone remodeling. Inhibition of osteoclast formation can greatly delay the progression of OA(9). Our previous study showed that TOD can dose-dependently inhibit osteoclast formation in vivo and inhibit osteoclast related genes, 
comprising NFATc1, Ctsk, Trap, Ctr, DC-STAMP, and VATPASE D2 in vitro. These outcomes demonstrate TOD is effective against bone loss.

The NF-KB and MAPK signalling pathways play important roles in chondrocyte inflammation and osteoclast formation $(48,49)$. Studies have shown that inhibition of both pathways can significantly improve the symptoms of OA and delay the progression of OA. Our results suggest that TOD inhibits NFKB and MAPK signalling by suppressing the phosphorylation of JNK, P65, P38, and ERK, which inhibits LPS-induced chondrocyte inflammation and RANKL-induced osteoclast formation.

ACLT of the knee joint is a classical mouse OA model $(50,51)$. In our study, we constructed the ACLT model, which had shown the similar pathological characteristics with those of human OA. Intraperitoneal injection of TOD significantly delayed the erosion of articular cartilage, reduced chondrocytes apoptosis, and inhibited osteoclast activity, thus delaying the progression of OA in mice. These results confirmed that TOD can be used as a potential agent for treating OA; however, whether TOD affects other targets to delay the progression of OA needs further study.

\section{Conclusion}

In summary, our results are the first to highlight that TOD, a kind of natural compound extracted from Toddalia asiatica (L.) Lam., inhibits matrix-degrading enzymes and pro-inflammatory cytokines secreted by chondrocytes to attenuate the progression of OA. TOD suppressed osteoclastogenesis through the suppression of the NF-KB and MAPK signalling paths. To study how TOD affects OA in vivo, we have constructed ACLT mouse OA model. The results confirmed that TOD delayed the erosion of articular cartilage, reduced the apoptosis of chondrocyte and inhibited the activity of osteoclasts, thus delaying $\mathrm{OA}$ progression in a mouse OA mode. These data for the first time implies that TOD can be a new therapeutic target for $O A$.

\section{Abbreviations}

$\mathrm{OA}$

Osteoarthritis

TOD

Toddalolactone

LPS

Lipopolysaccharides

BMMs

bone marrow derived macrophages

NF-KB

nuclear factor-kappaB

MAPK

mitogen-activated protein kinase 
MMPs

matrix metalloproteinases

ADAMTS

a disintegrin and metalloproteinase with thrombospondin motifs

DMSO

Dimethyl sulfoxide

TRAP

tartrate-resistant acid phosphate

BSA

Bovine serum albumin

M-CSF

macrophage colony stimulating factor

ACLT

anterior cruciate ligament transection

SMI

structure model index

Tb. Th

trabecular thickness

Tb.N

trabecular number

BMD

bone mineral density

$\mathrm{BV} / \mathrm{TV}$

bone volume/total tissue volume

Tb.Sp

trabecular separation.

\section{Declarations}

\section{Acknowledgements}

Not applicable

\section{Funding}

This work was financially supported by the National Natural Science Foundation of China (81871795 and 82172411), Shanghai Municipal Health and Family Planning Commission (SHDC12017121), Medical Engineering Cross Research Project of Shanghai Jiaotong University (YG2019QNB37) and Songjiang District Science and Technology Research project (18sjkjgg18).

\section{Availability of data and materials}


The data included in this investigation are available from the corresponding author.

\section{Declarations}

\section{Ethics approval and consent to participate}

All steps were implemented in accordance with animal welfare procedures and with permission from the Research Ethics Committee of Shanghai General Hospital (\#2021AW064).

\section{Consent for publication}

Not applicable.

\section{Competing interests}

The authors state that they have no conflict of interest.

\section{Authors' contributions}

Yiming Xu and Song Xue contributed to the concept and writing-original draft. Tian Zhang and Xinmeng Jin contributed to data curation and formal analysis. Yiming Xu, Tian Zhang, Xinmeng Jin, and Yiming Zhong contributed to analysis and interpretation of data. Yiming Xu, Song Xue, Hongjie Chen, Cong Wang, and Tian Zhang contributed to investigation and methodology. Weilin Sang, Xinmeng Jin, Haiming Lu, Libo Zhu, and Jinzhong Ma contributed to the visualization, resource, and project administration. Weilin Sang and Jinzhong Ma contributed to funding acquisition, conceptualization, supervision, and writing -review \& editing.

\section{References}

1. Bijlsma JWJ, Berenbaum F, Lafeber FPJG. Osteoarthritis: an update with relevance for clinical practice. The Lancet. 2011;377(9783):2115-26.

2. Dahaghin S, Bierma-Zeinstra SM, Ginai AZ, Pols HA, Hazes JM, Koes BW. Prevalence and pattern of radiographic hand osteoarthritis and association with pain and disability (the Rotterdam study). Ann Rheum Dis. 2005;64(5):682-7.

3. Abramoff B, Caldera FE. Osteoarthritis: Pathology, Diagnosis, and Treatment Options. Med Clin North Am. 2020;104(2):293-311.

4. Wu CL, Harasymowicz NS, Klimak MA, Collins KH, Guilak F. The role of macrophages in osteoarthritis and cartilage repair. Osteoarthritis Cartilage. 2020;28(5):544-54.

5. Crane JL, Cao X. Bone marrow mesenchymal stem cells and TGF-beta signaling in bone remodeling. J Clin Invest. 2014;124(2):466-72.

6. Zhao W, Wang T, Luo Q, Chen Y, Leung VY, Wen C, et al. Cartilage degeneration and excessive subchondral bone formation in spontaneous osteoarthritis involves altered TGF-beta signaling. $J$ 
Orthop Res. 2016;34(5):763-70.

7. Jiang Y, Zhu L, Zhang T, Lu H, Wang C, Xue B, et al. BRD4 has dual effects on the HMGB1 and NFkappaB signalling pathways and is a potential therapeutic target for osteoarthritis. Biochim Biophys Acta Mol Basis Dis. 2017;1863(12):3001-15.

8. Cai Z, Feng Y, Li C, Yang K, Sun T, Xu L, et al. Magnoflorine with hyaluronic acid gel promotes subchondral bone regeneration and attenuates cartilage degeneration in early osteoarthritis. Bone. 2018;116:266-78.

9. Goldring SR, Goldring MB. Changes in the osteochondral unit during osteoarthritis: structure, function and cartilage-bone crosstalk. Nat Rev Rheumatol. 2016;12(11):632-44.

10. Rahmati M, Nalesso G, Mobasheri A, Mozafari M. Aging and osteoarthritis: Central role of the extracellular matrix. Ageing Res Rev. 2017;40:20-30.

11. Batshon G, Elayyan J, Qiq O, Reich E, Ben-Aderet L, Kandel L, et al. Serum NT/CT SIRT1 ratio reflects early osteoarthritis and chondrosenescence. Ann Rheum Dis. 2020;79(10):1370-80.

12. Jeon $\mathrm{OH}$, Wilson DR, Clement CC, Rathod S, Cherry C, Powell B, et al. Senescence cell-associated extracellular vesicles serve as osteoarthritis disease and therapeutic markers. JCI Insight. 2019;4(7).

13. Hosseinzadeh A, Kamrava SK, Joghataei MT, Darabi R, Shakeri-Zadeh A, Shahriari M, et al. Apoptosis signaling pathways in osteoarthritis and possible protective role of melatonin. J Pineal Res. 2016;61(4):411-25.

14. Zhang G, Cao J, Yang E, Liang B, Ding J, Liang J, et al. Curcumin improves age-related and surgically induced osteoarthritis by promoting autophagy in mice. Biosci Rep. 2018;38(4).

15. Su W, Liu G, Liu X, Zhou Y, Sun Q, Zhen G, et al. Angiogenesis stimulated by elevated PDGF-BB in subchondral bone contributes to osteoarthritis development. JCI Insight. 2020;5(8).

16. Zheng CX, Sui BD, Qiu XY, Hu CH, Jin Y. Mitochondrial Regulation of Stem Cells in Bone Homeostasis. Trends Mol Med. 2020;26(1):89-104.

17. Siddiqui JA, Partridge NC. Physiological Bone Remodeling: Systemic Regulation and Growth Factor Involvement. Physiology (Bethesda). 2016;31(3):233-45.

18. Rigoglou S, Papavassiliou AG. The NF-kappaB signalling pathway in osteoarthritis. Int J Biochem Cell Biol. 2013;45(11):2580-4.

19. Mlost J, Kostrzewa M, Borczyk M, Bryk M, Chwastek J, Korostynski M, et al. CB2 agonism controls pain and subchondral bone degeneration induced by mono-iodoacetate: Implications GPCR functional bias and tolerance development. Biomed Pharmacother. 2021;136:111283.

20. Zhu S, Zhu J, Zhen G, Hu Y, An S, Li Y, et al. Subchondral bone osteoclasts induce sensory innervation and osteoarthritis pain. J Clin Invest. 2019;129(3):1076-93.

21. Gu J, Lin H, Zhang Y, Xu T, Wang T, Xue X, et al. Activation of GPR40 Suppresses AGE-Induced Reduction of Type II Collagen and Aggrecan in Human SW1353 Chondrocytes. Drug Des Devel Ther. 2020;14:2371-9. 
22. Choi DJ, Choi SI, Choi BR, Lee YS, Lee DY, Kim GS. Cartilage protective and anti-analgesic effects of ALM16 on monosodium iodoacetate induced osteoarthritis in rats. BMC Complement Altern Med. 2019;19(1):325.

23. Li H, Chen J, Li B, Fang X. The protective effects of dulaglutide against advanced glycation end products (AGEs)-induced degradation of type collagen and aggrecan in human SW1353 chondrocytes. Chem Biol Interact. 2020;322:108968.

24. Kong L, Smith W, Hao D. Overview of RAW264.7 for osteoclastogensis study: Phenotype and stimuli. J Cell Mol Med. 2019;23(5):3077-87.

25. Huang J, Yin H, Rao SS, Xie PL, Cao X, Rao T, et al. Harmine enhances type H vessel formation and prevents bone loss in ovariectomized mice. Theranostics. 2018;8(9):2435-46.

26. Jiang Y, Sang W, Wang C, Lu H, Zhang T, Wang Z, et al. Oxymatrine exerts protective effects on osteoarthritis via modulating chondrocyte homoeostasis and suppressing osteoclastogenesis. J Cell Mol Med. 2018.

27. Meng J, Zhang W, Wang C, Zhang W, Zhou C, Jiang G, et al. Catalpol suppresses osteoclastogenesis and attenuates osteoclast-derived bone resorption by modulating PTEN activity. Biochem Pharmacol. 2020;171:113715.

28. Ni J, Zhao Y, Su J, Liu Z, Fang S, Li L, et al. Toddalolactone Protects Lipopolysaccharide-Induced Sepsis and Attenuates Lipopolysaccharide-Induced Inflammatory Response by Modulating HMGB1NF-kappaB Translocation. Front Pharmacol. 2020;11:109.

29. Yu B, Zhang G, Jin L, Zhang B, Yan D, Yang H, et al. Inhibition of PAl-1 Activity by Toddalolactone as a Mechanism for Promoting Blood Circulation and Removing Stasis by Chinese Herb Zanthoxylum nitidum var. tomentosum. Front Pharmacol. 2017;8:489.

30. Reinhardt JK, Zimmermann-Klemd AM, Danton O, Smiesko M, Grundemann C, Hamburger M. Compounds from Toddalia asiatica: Immunosuppressant Activity and Absolute Configurations. J Nat Prod. 2020;83(10):3012-20.

31. Kapoor M, Martel-Pelletier J, Lajeunesse D, Pelletier JP, Fahmi H. Role of proinflammatory cytokines in the pathophysiology of osteoarthritis. Nat Rev Rheumatol. 2011;7(1):33-42.

32. Park KR, Yun HM. RANKL-induced osteoclastogenesis in bone marrow-derived macrophages is suppressed by cisapride. Toxicology. 2019;422:95-101.

33. Saito T, Tanaka S. Molecular mechanisms underlying osteoarthritis development: Notch and NFkappaB. Arthritis Res Ther. 2017;19(1):94.

34. Wong SK, Chin KY, Ima-Nirwana S. Berberine and musculoskeletal disorders: The therapeutic potential and underlying molecular mechanisms. Phytomedicine. 2020;73:152892.

35. Zhou F, Mei J, Han X, Li H, Yang S, Wang M, et al. Kinsenoside attenuates osteoarthritis by repolarizing macrophages through inactivating NF-kappaB/MAPK signaling and protecting chondrocytes. Acta Pharm Sin B. 2019;9(5):973-85.

36. Wu Y, Wang Z, Fu X, Lin Z, Yu K. Geraniol-mediated osteoarthritis improvement by down-regulating PI3K/Akt/NF-kappaB and MAPK signals: In vivo and in vitro studies. Int Immunopharmacol. 
2020;86:106713.

37. Glasson SS, Blanchet TJ, Morris EA. The surgical destabilization of the medial meniscus (DMM) model of osteoarthritis in the 129/SvEv mouse. Osteoarthritis Cartilage. 2007;15(9):1061-9.

38. Ahmad N, Ansari MY, Haqqi TM. Role of iNOS in osteoarthritis: Pathological and therapeutic aspects. J Cell Physiol. 2020;235(10):6366-76.

39. Hwang HS, Kim HA. Chondrocyte Apoptosis in the Pathogenesis of Osteoarthritis. Int J Mol Sci. 2015;16(11):26035-54.

40. Jamshidi A, Pelletier JP, Martel-Pelletier J. Machine-learning-based patient-specific prediction models for knee osteoarthritis. Nat Rev Rheumatol. 2019;15(1):49-60.

41. Makris EA, Gomoll AH, Malizos KN, Hu JC, Athanasiou KA. Repair and tissue engineering techniques for articular cartilage. Nat Rev Rheumatol. 2015;11(1):21-34.

42. De Bari C, Roelofs AJ. Stem cell-based therapeutic strategies for cartilage defects and osteoarthritis. Curr Opin Pharmacol. 2018;40:74-80.

43. Rim YA, Nam Y, Ju JH. The Role of Chondrocyte Hypertrophy and Senescence in Osteoarthritis Initiation and Progression. Int J Mol Sci. 2020;21(7).

44. Kovacs B, Vajda E, Nagy EE. Regulatory Effects and Interactions of the Wnt and OPG-RANKL-RANK Signaling at the Bone-Cartilage Interface in Osteoarthritis. Int J Mol Sci. 2019;20(18).

45. Burr DB, Gallant MA. Bone remodelling in osteoarthritis. Nat Rev Rheumatol. 2012;8(11):665-73.

46. Goldring SR. Role of bone in osteoarthritis pathogenesis. Med Clin North Am. 2009;93(1):25-35, xv.

47. Goldring MB, Goldring SR. Articular cartilage and subchondral bone in the pathogenesis of osteoarthritis. Ann N Y Acad Sci. 2010;1192:230-7.

48. Ding Y, Wang L, Zhao Q, Wu Z, Kong L. MicroRNA93 inhibits chondrocyte apoptosis and inflammation in osteoarthritis by targeting the TLR4/NFkappaB signaling pathway. Int J Mol Med. 2019;43(2):779-90.

49. Wang C, Gao Y, Zhang Z, Chi Q, Liu Y, Yang L, et al. Safflower yellow alleviates osteoarthritis and prevents inflammation by inhibiting PGE2 release and regulating NF-kappaB/SIRT1/AMPK signaling pathways. Phytomedicine. 2020;78:153305.

50. Faust $\mathrm{HJ}$, Zhang H, Han J, Wolf MT, Jeon OH, Sadtler K, et al. IL-17 and immunologically induced senescence regulate response to injury in osteoarthritis. J Clin Invest. 2020;130(10):5493-507.

51. Tong W, Zeng Y, Chow DHK, Yeung W, Xu J, Deng Y, et al. Wnt16 attenuates osteoarthritis progression through a PCP/JNK-mTORC1-PTHrP cascade. Ann Rheum Dis. 2019;78(4):551-61.

\section{Figures}


A

B<smiles>COc1cc2oc(=O)ccc2c(OC)c1CC(O)C(C)(C)O</smiles>

C

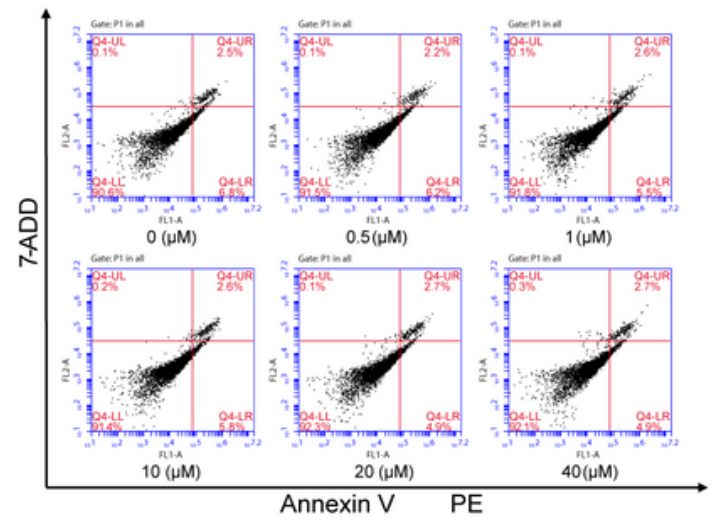

SW1353

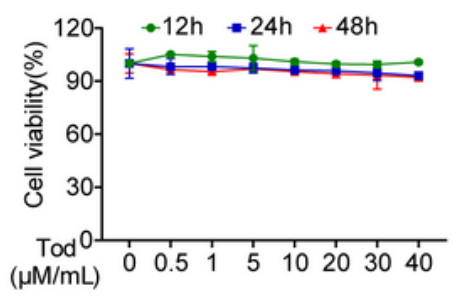

$\mathrm{D}$

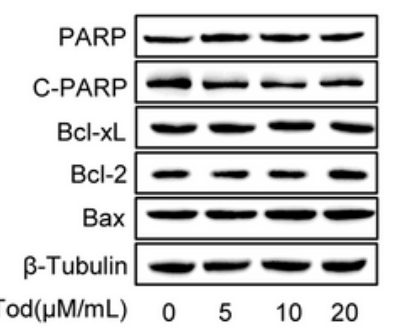

$\mathrm{E}$
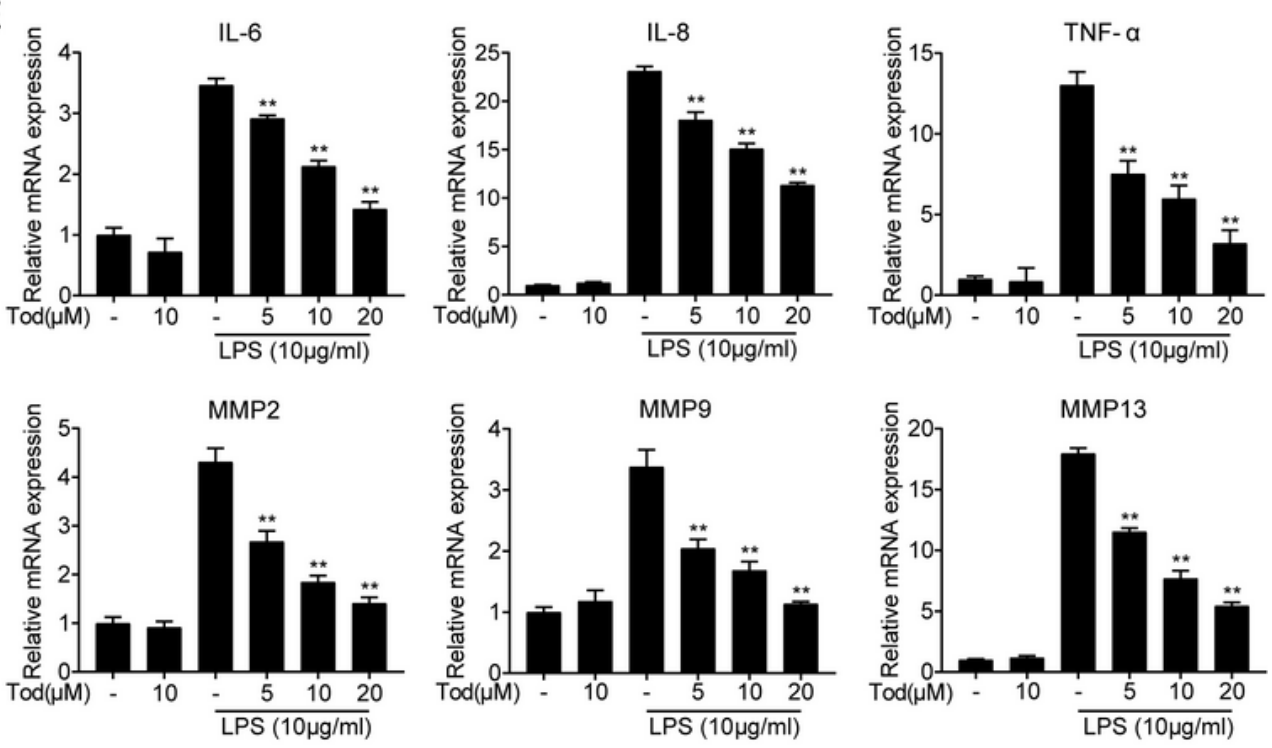

$\mathrm{F}$

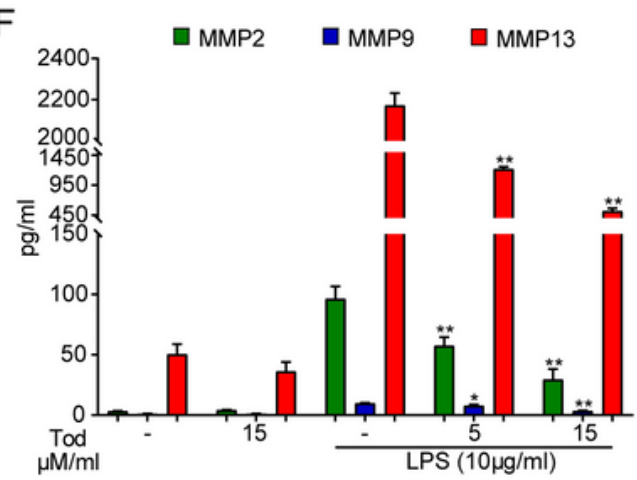

- TNF-a $\quad$ IL-6

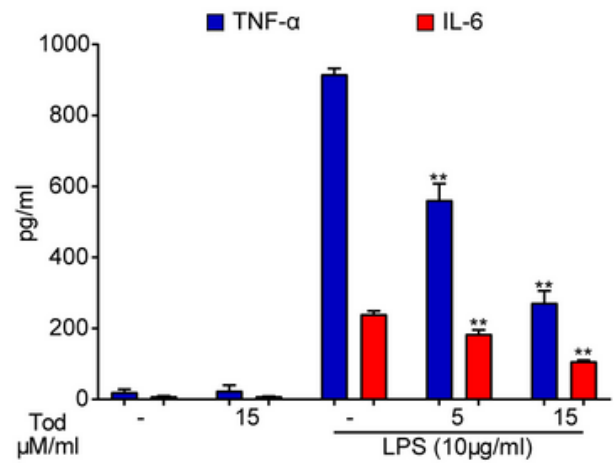

Figure 1

TOD inhibited the expression of inflammatory and catabolic mediators in IL-1 $\beta$ induced chondrocytes. A Chemical structure of TOD. B The effect of TOD on the cell viability of SW1353 was evaluated by CCK-8. C Gradient concentrations $(0,0.5,1,5,10,20,40 \mu \mathrm{M} / \mathrm{ml})$ of TOD on the apoptosis level of SW1353 was measured by flow cytometry. D Western blotting performed after SW1353 cells were treated with gradient concentrations $(0,5,10$, and $20 \mu \mathrm{M} / \mathrm{ml})$ of TOD; $\beta$-Tubulin was used as the loading control. E Pro- 
inflammatory cytokines, including IL-6, IL-8, TNF-a, MMP2, MMP9, and MMP13 were measured by RTPCR. F ELISA was used to measure the expression level of MMP2, MMP9, MMP13, IL-6, and TNF-a. Data represent means \pm SD of triplicate independent experiments. ${ }^{*}{ }^{*}$ indicates $p<0.05,{ }^{* *}$, \#\# indicates $p<0.01$, ns indicates not significant.
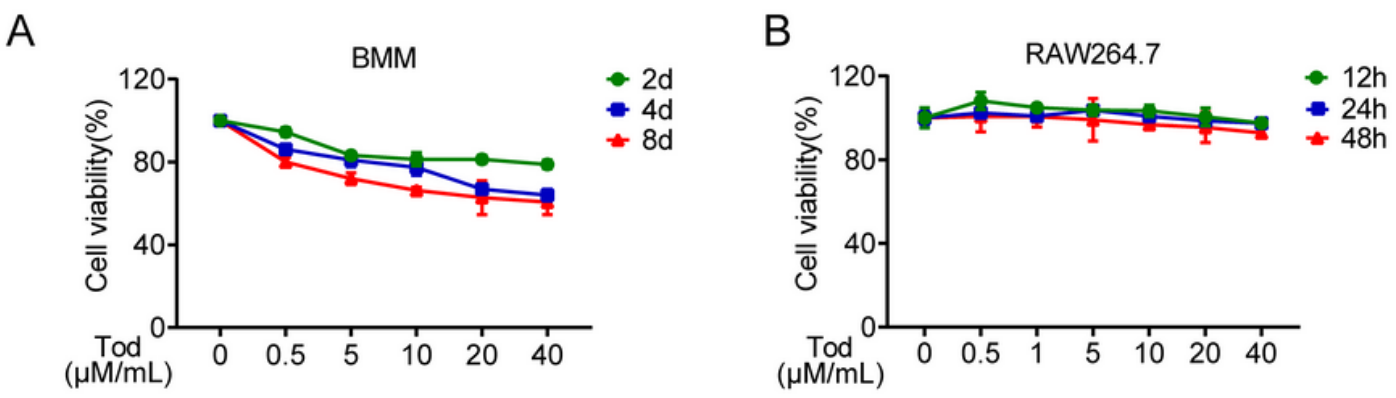

C

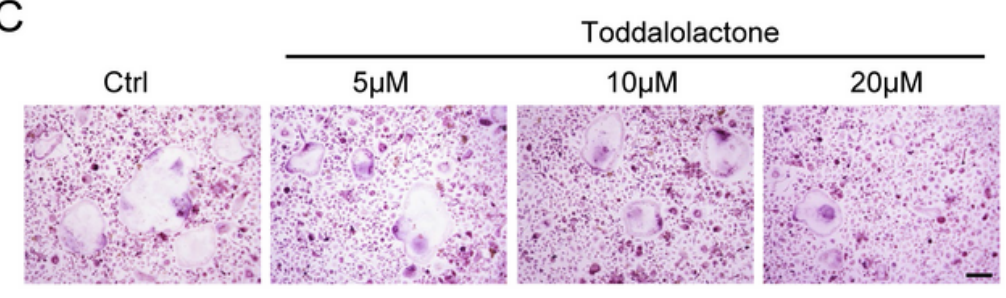

$\mathrm{E}$

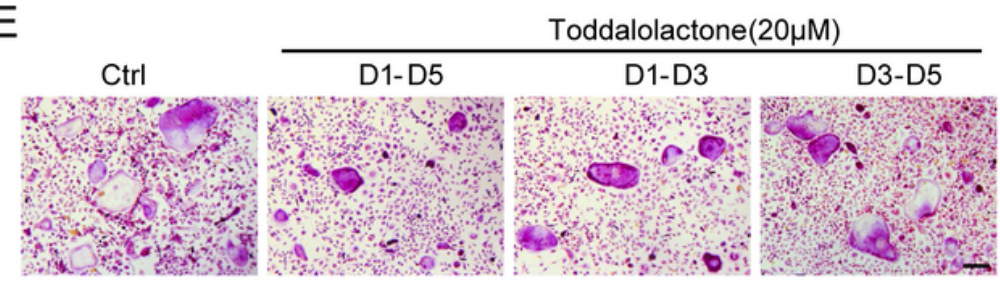

G
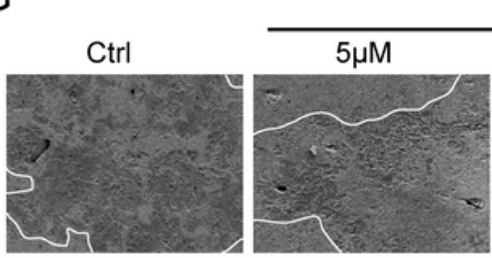

Toddalolactone
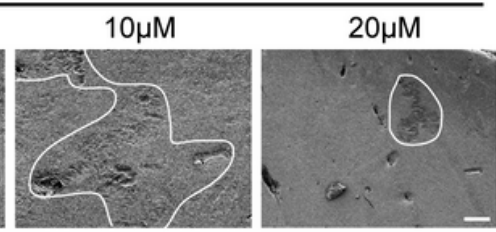

I
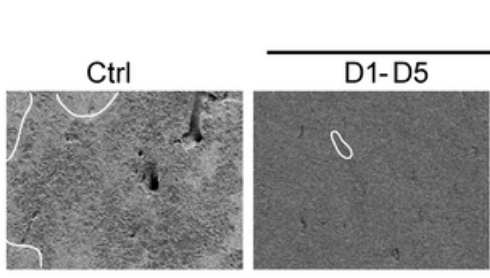

Toddalolactone $(20 \mu \mathrm{M})$

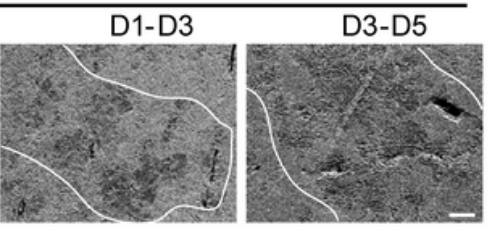

D
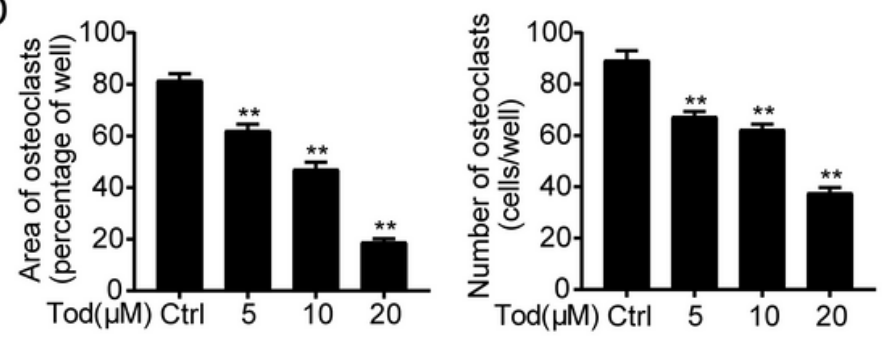

$\mathrm{F}$
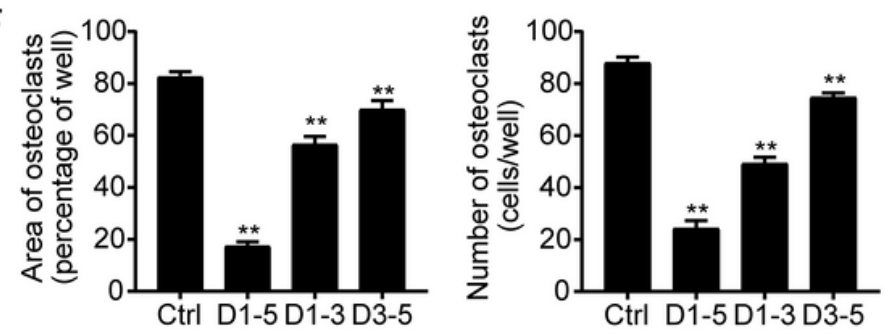

$\mathrm{H}$
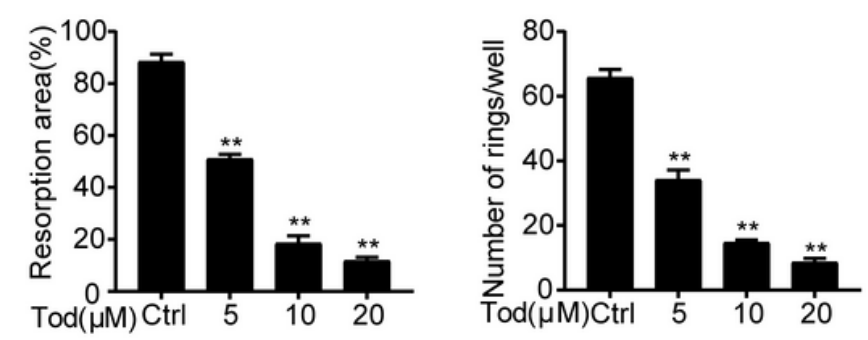

$\mathrm{J}$
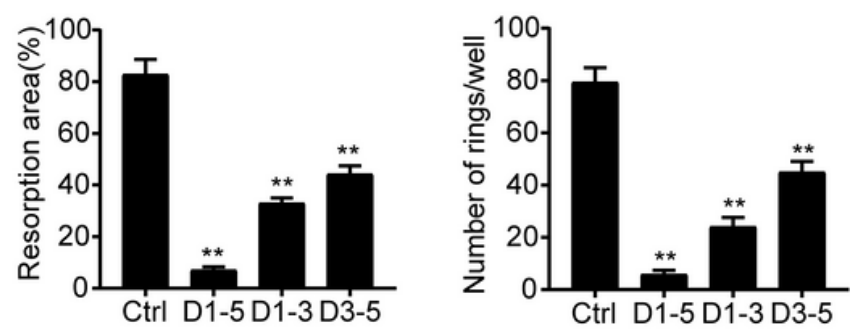

Figure 2

TOD inhibits RANKL-induced osteoclast differentiation and bone resorption without cytotoxicity in vitro. A The cell viability of BMMs exposed to TOD was measured by CCK-8. B The cell viability of RAW264.7 cells exposed to TOD was assessed by CCK-8. C BMMs were cultured with M-CSF ( $25 \mathrm{ng} / \mathrm{ml}$ ), RANKL (50 
$\mathrm{ng} / \mathrm{ml})$, and the indicated concentrations $(0,5,10$, and $20 \mu \mathrm{M})$ of TOD for 5 days. The effect of TOD on BMMs differentiation was detected using TRAP staining. Scale bar: $100 \mu \mathrm{m}$. D The area and number of osteoclasts were quantified per well. E BMMs were cultured with M-CSF $(25 \mathrm{ng} / \mathrm{ml})$ and RANKL (50 $\mathrm{ng} / \mathrm{ml})$ for 5 days, and TOD $(20 \mu \mathrm{M})$ was added at different stages during osteoclast differentiation. The effect of TOD on BMMs differentiation was detected using TRAP staining. Scale bar: $100 \mu \mathrm{m}$. F The area and number of osteoclasts were quantified per well. G BMMs were cultured with M-CSF $(25 \mathrm{ng} / \mathrm{ml})$, RANKL $(50 \mathrm{ng} / \mathrm{ml})$, and the indicated concentrations $(0,5,10$, and $20 \mu \mathrm{M})$ of TOD for 5 days. Scanning electron microscopy was used to observe bone resorption pits. Scale bar: $100 \mu \mathrm{m}$. $\mathbf{H}$ The resorption area and number of rings were quantified. I BMMs were cultured with M-CSF $(25 \mathrm{ng} / \mathrm{ml})$ and RANKL (50 $\mathrm{ng} / \mathrm{ml})$ for 5 days, and TOD $(20 \mu \mathrm{M})$ was added at different stages during osteoclast differentiation. Scanning electron microscopy was used to observe bone resorption pits. Scale bar: $100 \mu \mathrm{m}$. J The resorption area and number of rings were quantified. Data represent means \pm SD of triplicate independent experiments. ${ }^{*}{ }^{*}$ indicates $p<0.05,{ }^{* *}, \#$ indicates $p<0.01,{ }^{\text {ns }}$ indicates not significant. 
A
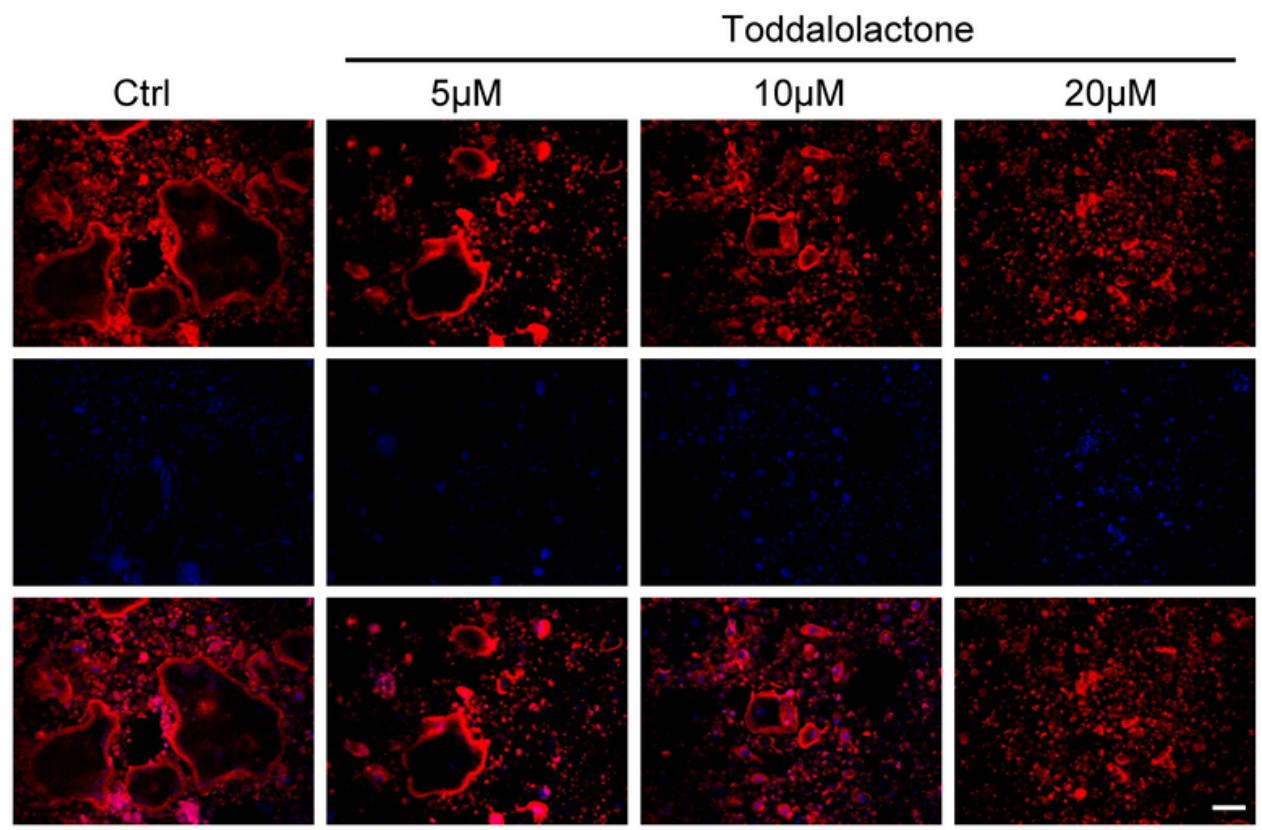

C
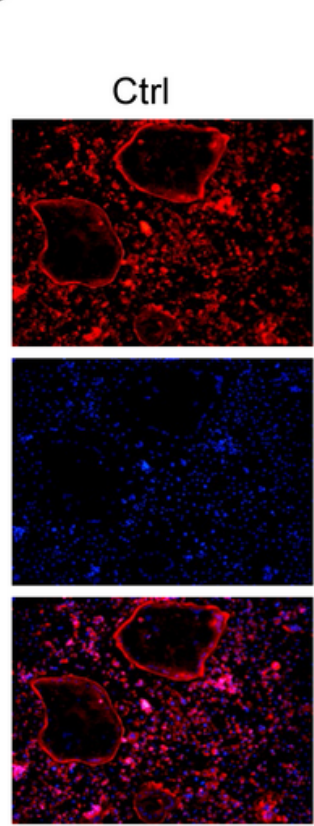

Toddalolactone
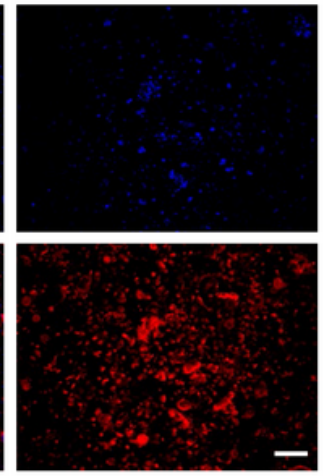

Toddalolactone $(20 \mu \mathrm{M})$
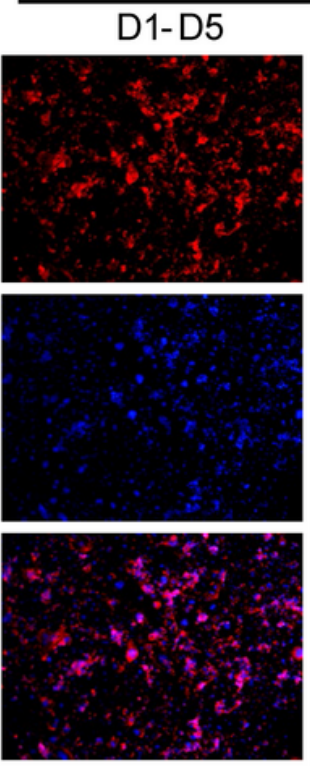

B
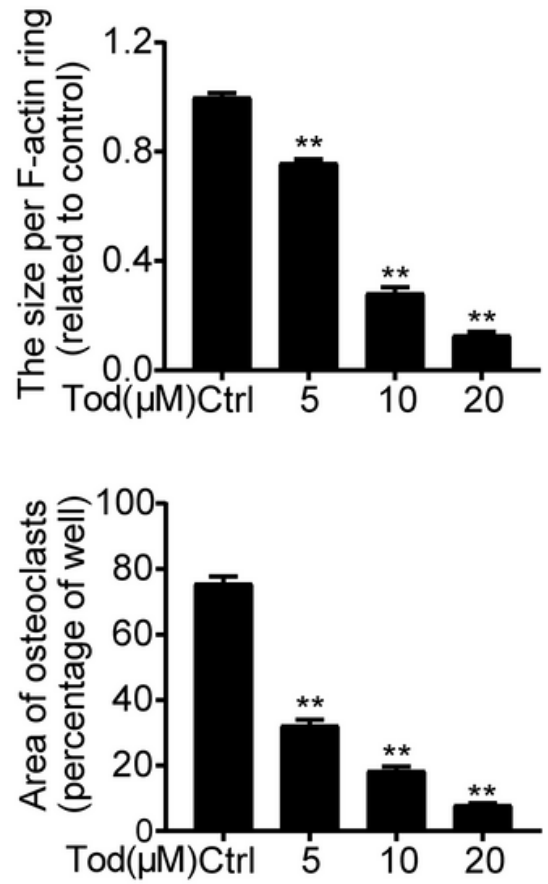

D
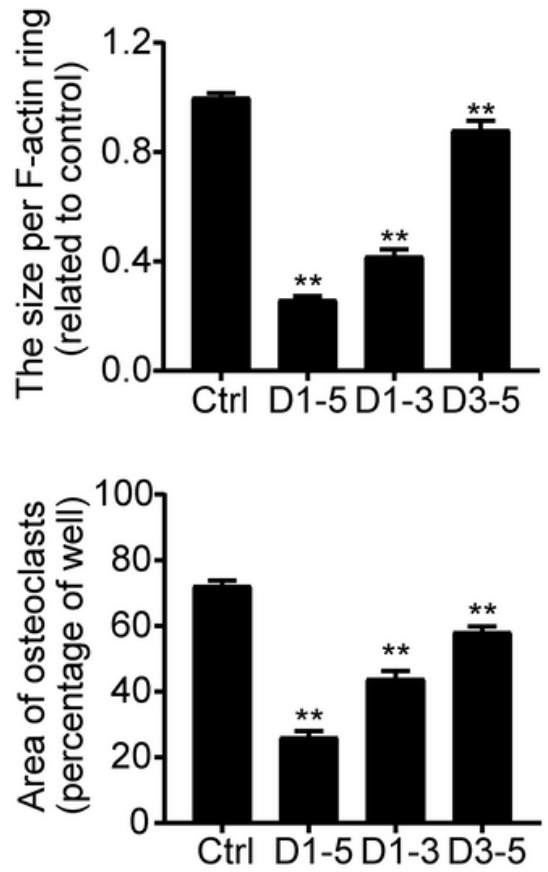

Figure 3

TOD inhibits the formation of F-actin ring. A BMMs were cultured with M-CSF ( $25 \mathrm{ng} / \mathrm{ml})$, RANKL (50 $\mathrm{ng} / \mathrm{ml})$, and the indicated concentrations $(0,5,10$, and $20 \mu \mathrm{M})$ of TOD for 5 days. After differentiation, BMMs were fixed and stained for F-actin ring. Scale bar: $100 \mu \mathrm{m}$. B The size per F-actin ring and area of osteoclasts were quantified. C BMMs were cultured with M-CSF $(25 \mathrm{ng} / \mathrm{ml})$ and RANKL $(50 \mathrm{ng} / \mathrm{ml})$ for 5 days, and TOD $(20 \mu \mathrm{M})$ was added at different stages during osteoclast differentiation. After differentiation, BMMs were fixed and stained for F-actin ring. Scale bar: $100 \mu \mathrm{m}$. D The size per F-actin 
ring and area of osteoclasts were quantified. Data represent means \pm SD of triplicate independent experiments. ${ }^{*}, \#_{\text {indicates }} \mathrm{p}<0.05,{ }^{\star *}, \# \#_{\text {indicates }} \mathrm{p}<0.01,{ }^{\mathrm{ns}}$ indicates not significant.

A
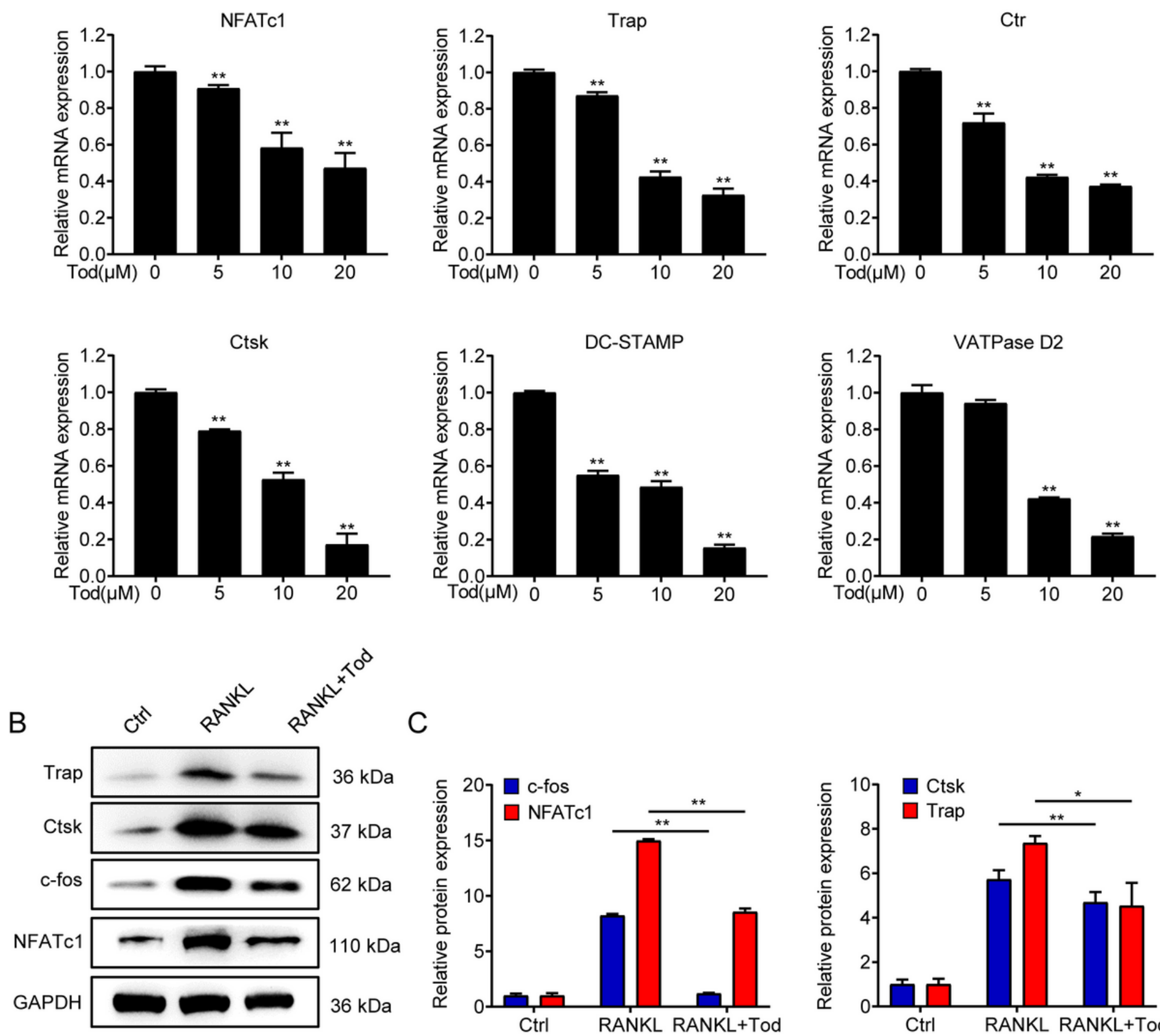

C
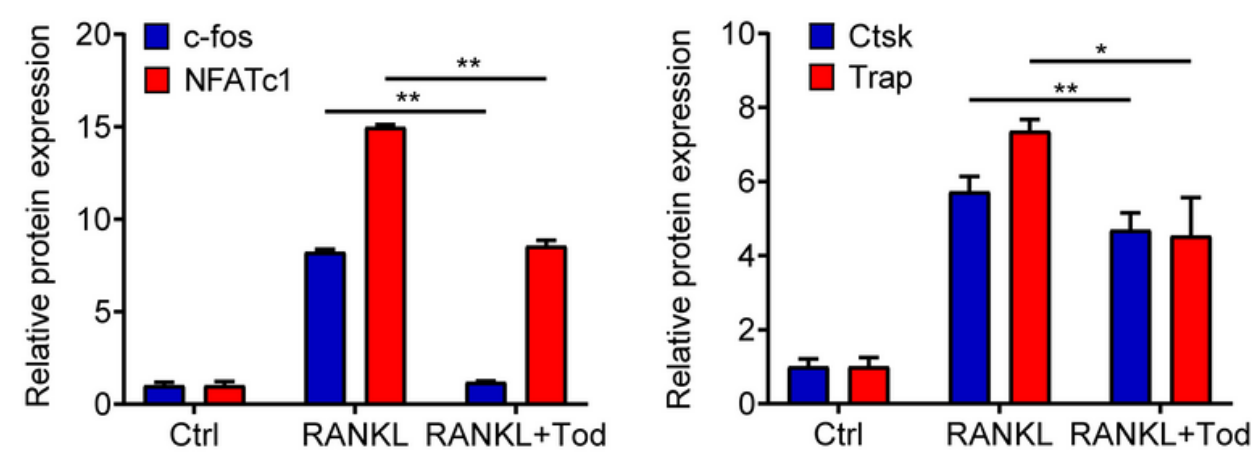

Figure 4

TOD suppresses RANKL-induced gene expression during osteoclastogenesis in vitro. A mRNA expression of osteoclast marker genes (NFATc1, Trap, Ctr, Ctsk, DC-STAMP, and VATPase D2) were detected by RTPCR. B Western blotting performed that TOD suppressed the activation of osteoclast related protein (Trap, Ctsk, c-Fos, and NFATc1); GAPDH was used as the loading control. C ImageJ software was used to quantify the density of the western blot bands shown in B. Data represent means \pm SD of triplicate independent experiments. ${ }^{*},{ }_{i}$ indicates $p<0.05,{ }^{* *}, \#$ indicates $p<0.01,{ }^{n s}$ indicates not significant. 
A

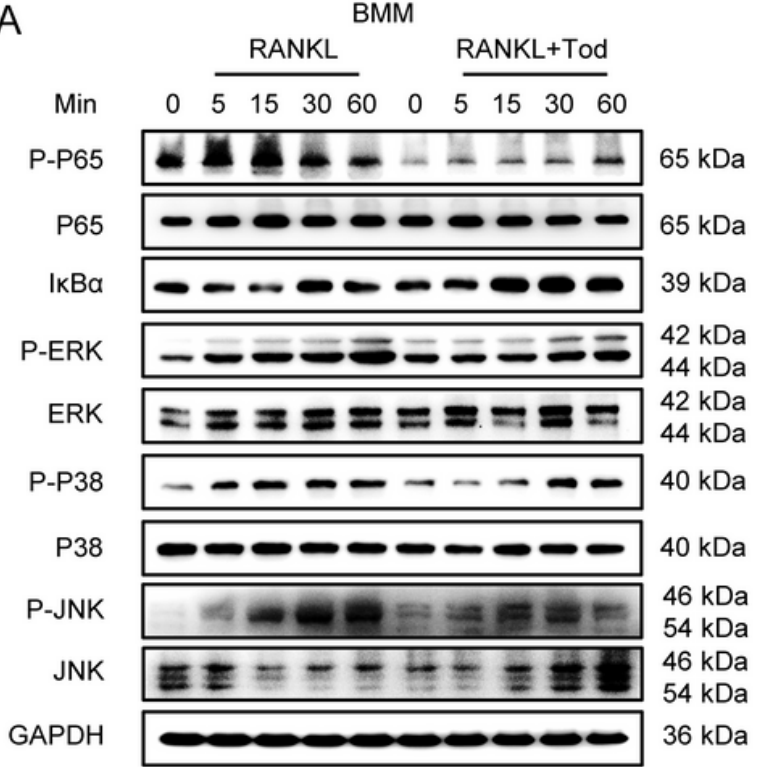

C

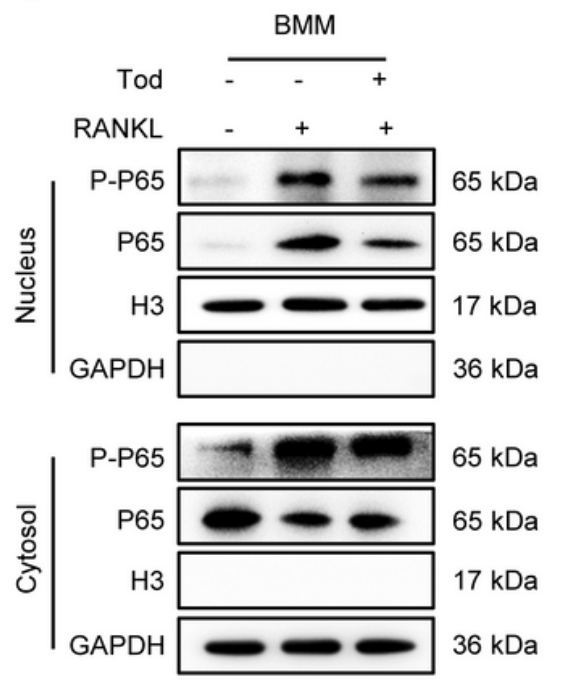

D
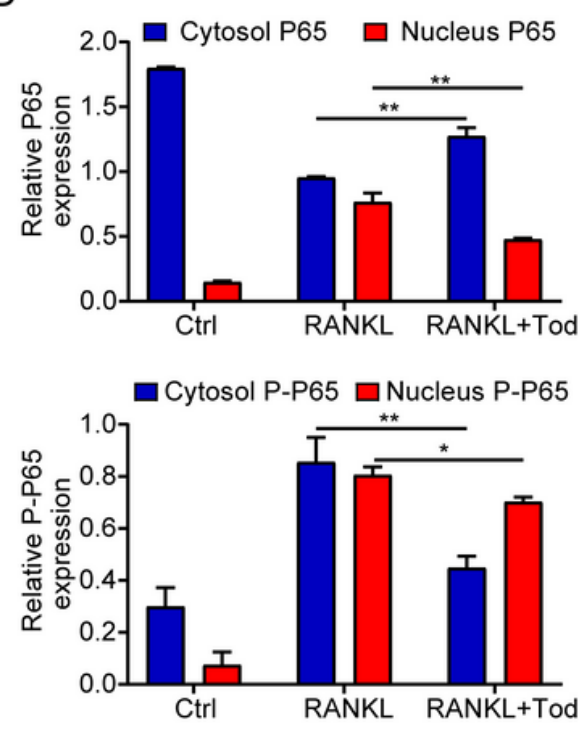

B
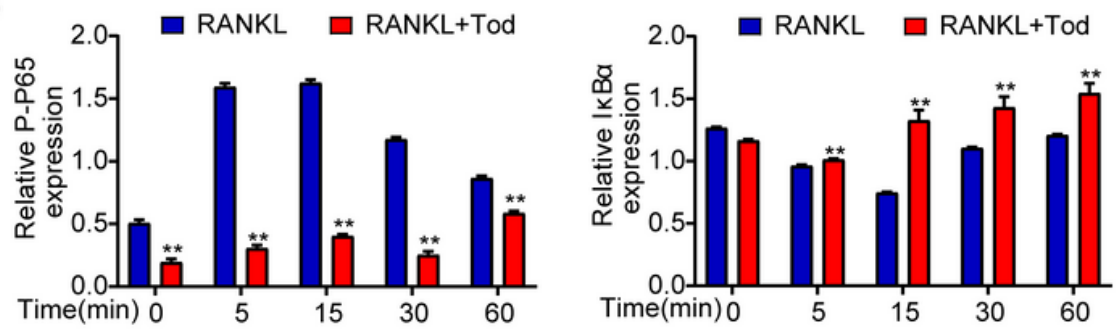

E
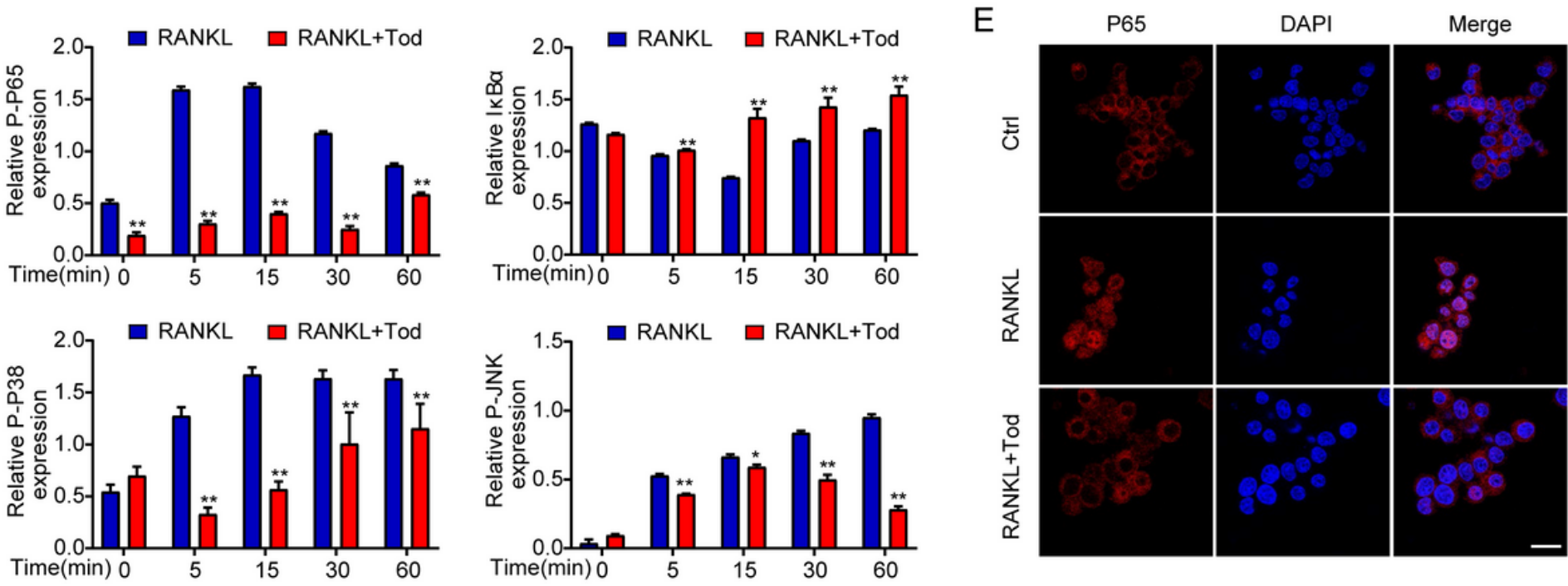

Figure 5

TOD inhibits the activation of the NF-KB and MAPK signalling pathways in SW1353 cells. A Western blotting performed the activity of NF-KB and MAPK signalling pathways in SW1353 cells; GAPDH was used as the loading control. B ImageJ software was used to quantify the density of the western blot bands (P-P65, IKBa, P-P38, and P-JNK) shown in A. C Western blots performed the protein expression of P65 in the nucleus and cytoplasm in SW1353 cells. D ImageJ software was used to quantify the density of the western blot bands in C. E Immunofluorescence was used to determine nuclear translocation of p65 in SW1353 cells. Scale bar: $100 \mu \mathrm{m}$. Data represent means \pm SD of triplicate independent experiments. ${ }^{*}$, indicates $p<0.05,{ }^{* *}, \#$ indicates $p<0.01$, ns indicates not significant. 
A

SW1353

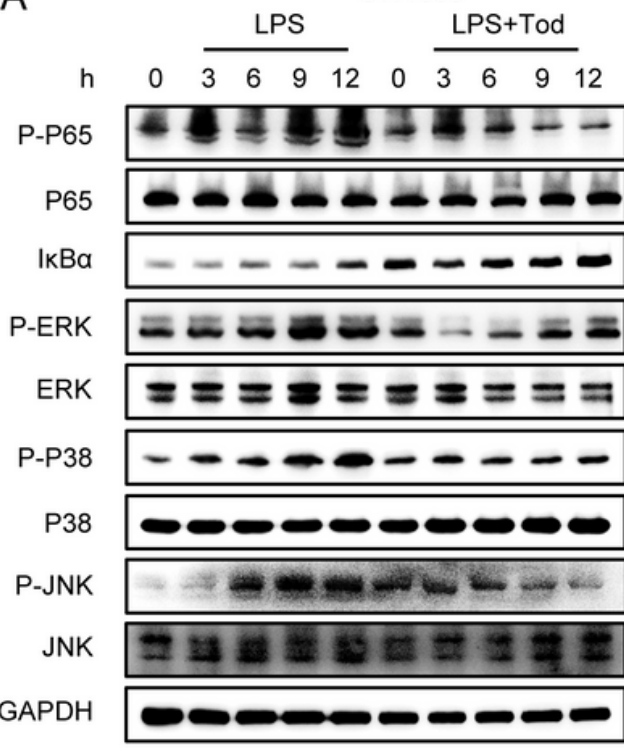

$5 \mathrm{kDa}$

$65 \mathrm{kDa}$

$39 \mathrm{kDa}$

$42 \mathrm{kDa}$

$44 \mathrm{kDa}$

$42 \mathrm{kDa}$ $44 \mathrm{kDa}$

$40 \mathrm{kDa}$

$40 \mathrm{kDa}$

$46 \mathrm{kDa}$

$54 \mathrm{kDa}$

$46 \mathrm{kDa}$

$54 \mathrm{kDa}$

$36 \mathrm{kDa}$
C

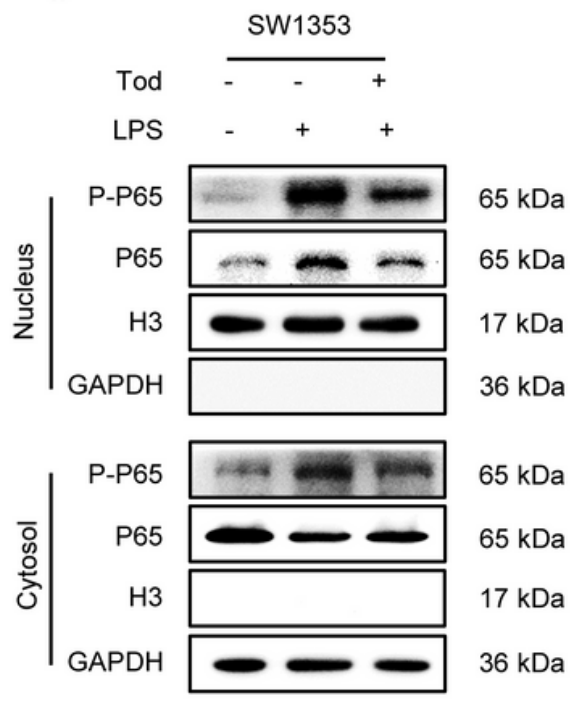

D
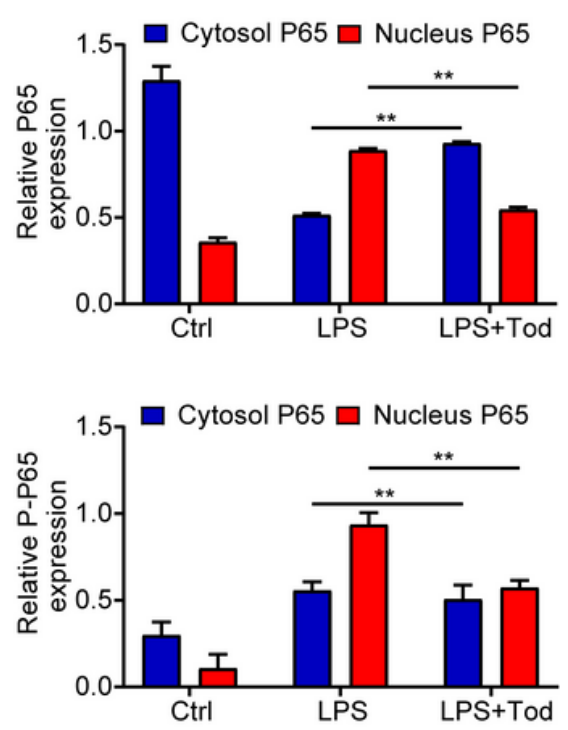

B
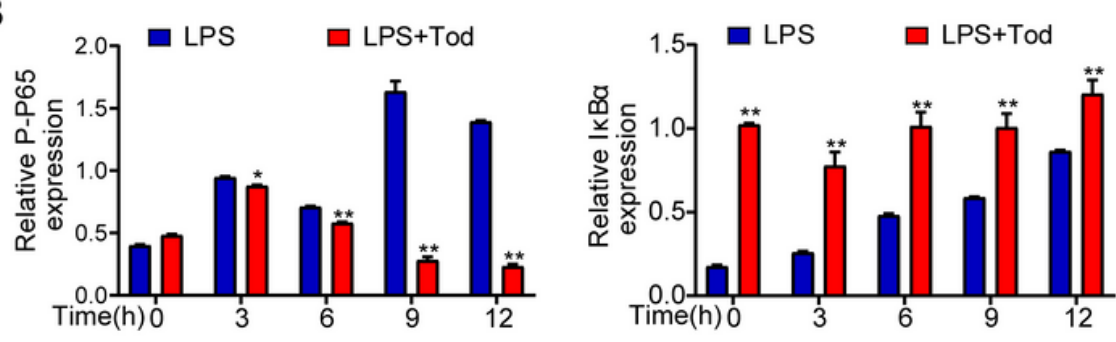

E

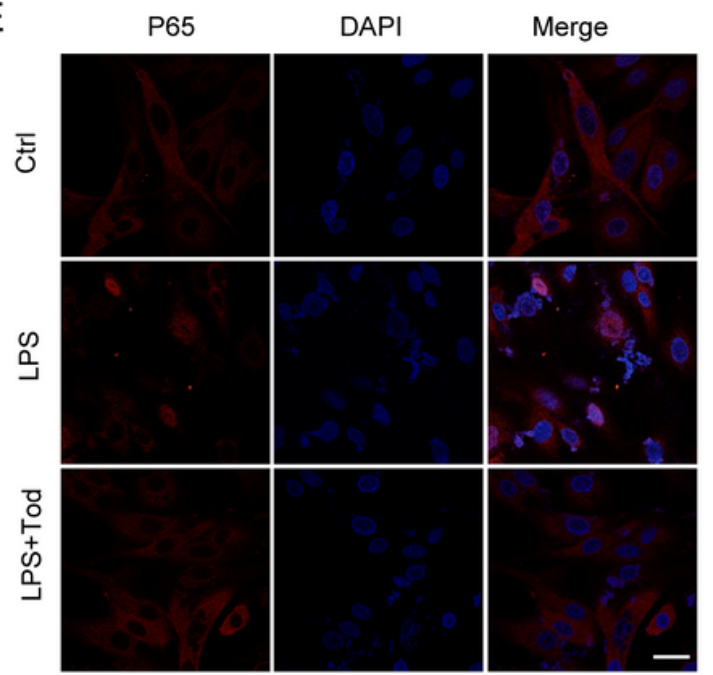

Figure 6

TOD attenuates the activation of the NF-KB and MAPK signalling pathways in BMMs. A Western blotting performed the activity of NF-KB and MAPK signalling pathways in RAW264.7 cells; GAPDH was used as the loading control. B ImageJ software was used to quantify the density of the western blot bands ( $P$ P65, ІкBa, P-P38, and P-JNK) shown in A. C Western blots performed the protein expression of P65 in the nucleus and cytoplasm in RAW264.7 cells. D ImageJ software was used to quantify the density of the western blot bands in C. E Immunofluorescence was used to determine nuclear translocation of p65 in RAW264.7 cells. Scale bar: $100 \mu \mathrm{m}$. Data represent means \pm SD of triplicate independent experiments. * \#indicates $p<0.05,{ }^{* *}, \# \#_{\text {indicates }}$ p $<0.01,{ }^{n s}$ indicates not significant. 


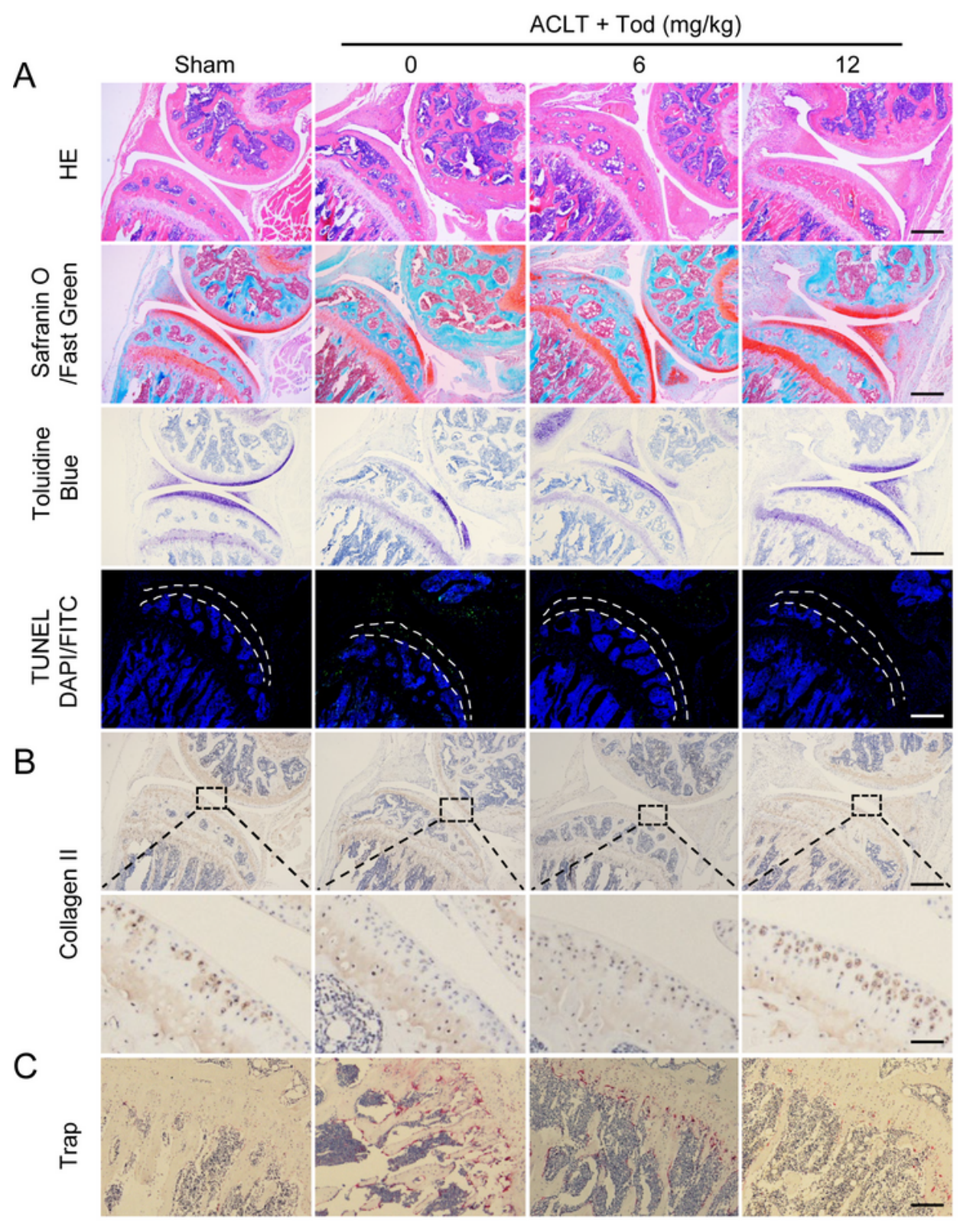

D

$\mathrm{E}$
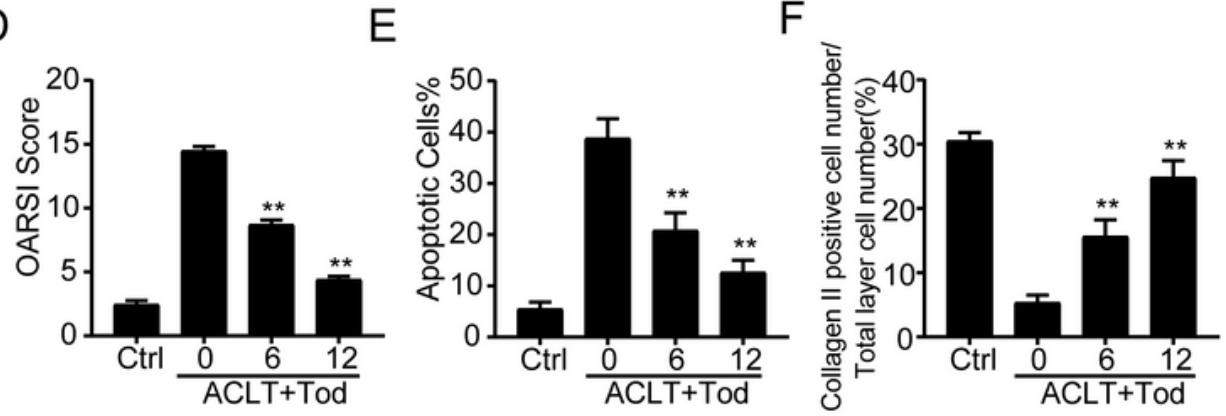

Figure 7

TOD protects against ACLT-induced cartilage destruction and inhibits osteoclastogenesis in vivo. A H\&E staining, Safranin O/Fast Green staining, Toluidine Blue, and TUNEL DAPI/FITC staining were performed respectively. Scale bar: $100 \mu \mathrm{m}$. B Immunohistochemical analysis of Collagen II. Scale bar: $100 \mu \mathrm{m}$ (upper); $10 \mu \mathrm{m}$ (lower). C TRAP staining of the osteoclasts in the subchondral plate. Scale bar: $200 \mu \mathrm{m}$. D The OARSI scores of different groups were assessed. $\mathrm{E}$ The percentage of apoptotic chondrocytes in 
cartilage was calculated. F Quantitative analysis of the immunohistochemical staining of Collagen II. Data represent means \pm SD of triplicate independent experiments. ${ }^{*}{ }^{*}$ indicates $p<0.05,{ }^{* *}, \#{ }$ indicates $p<$ $0.01,{ }^{n s}$ indicates not significant.

A $\mathrm{ACLT}+\mathrm{Tod}(\mathrm{mg} / \mathrm{kg})$
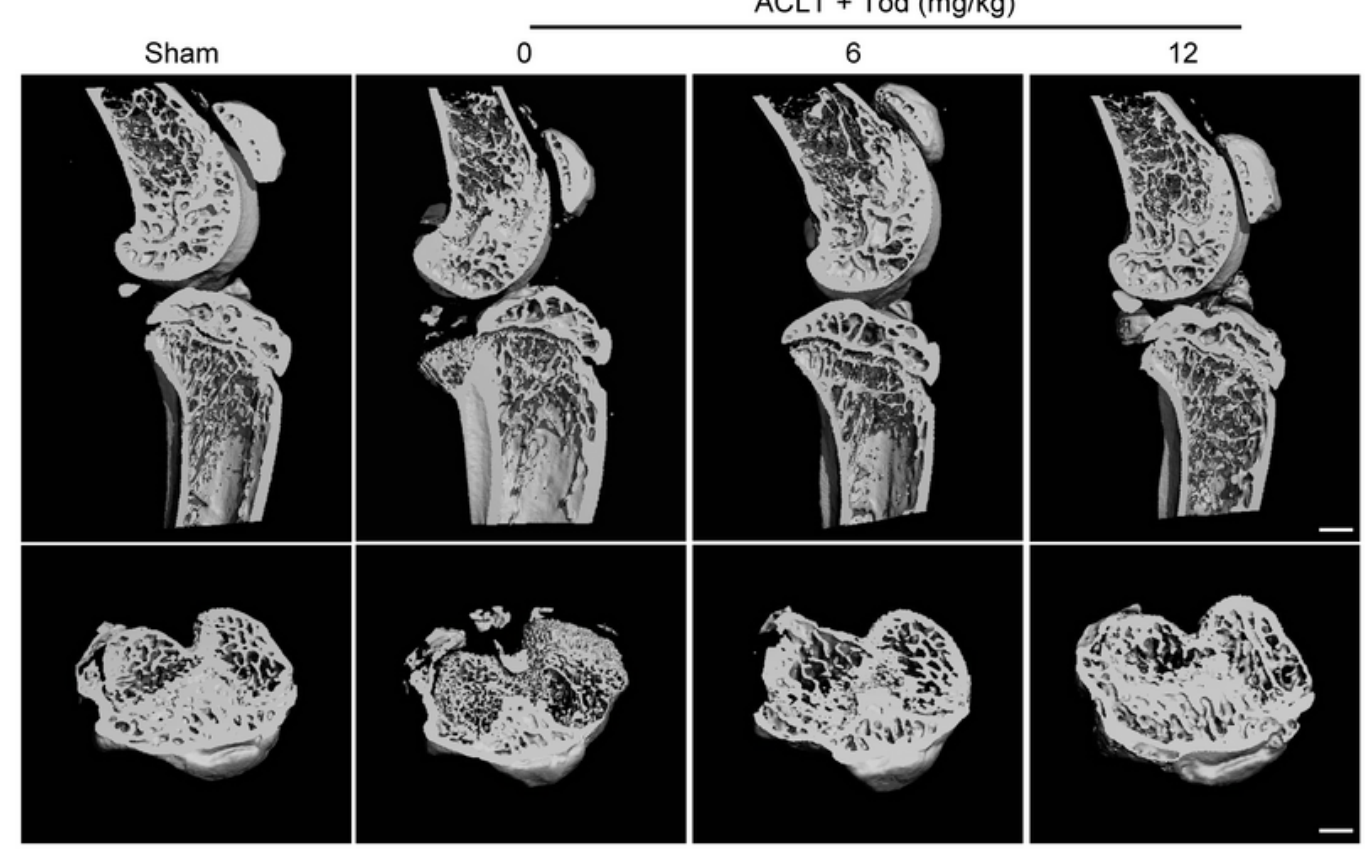

B
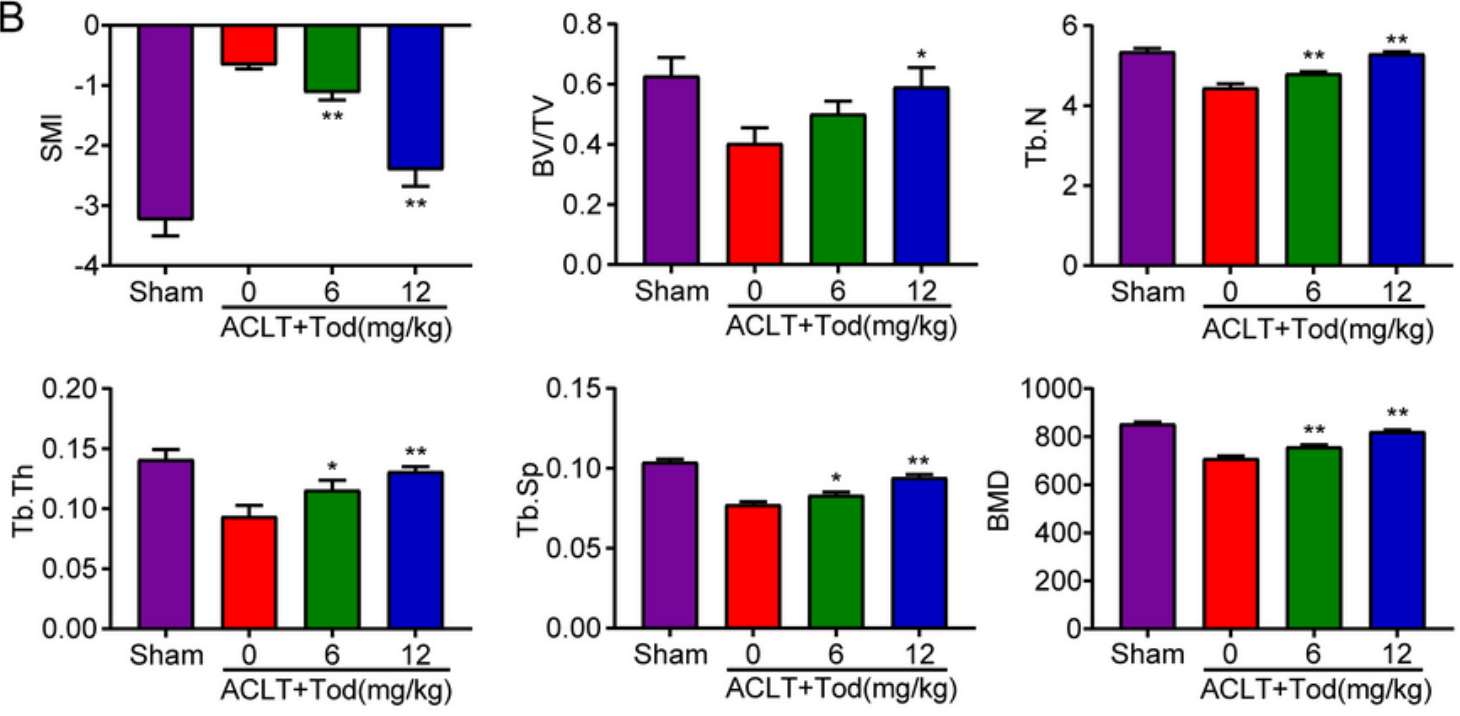

C
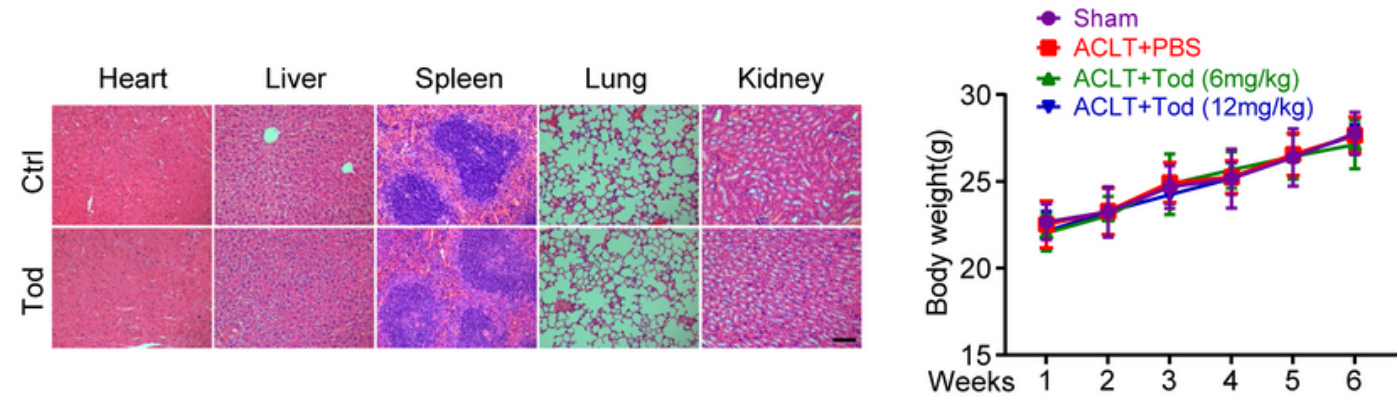

Figure 8 
TOD protects against ACLT-induced bone loss in vivo. A 3D reconstruction micro-CT images were performed for each group. Scale bar: $2 \mathrm{~mm}$. B Quantification of SMI, BV/TV, Tb.Th, Tb.Sp, Tb.N, and BMD. C H\&E staining of important organs. Scale bar: $200 \mu \mathrm{m}$; Body weights were recorded once per week. Data represent means \pm SD of triplicate independent experiments. ${ }^{*}{ }^{*}$ indicates $p<0.05,{ }^{*},{ }^{*}{ }^{\text {indicates }} p<$ $0.01,{ }^{n s}$ indicates not significant. 